\title{
Classical Negation and Game-Theoretical Semantics
}

\author{
Tero Tulenheimo
}

\begin{abstract}
Typical applications of Hintikka's game-theoretical semantics (GTS) give rise to semantic attributes-truth, falsity-expressible in the $\Sigma_{1}^{1}$-fragment of second-order logic. Actually a much more general notion of semantic attribute is motivated by strategic considerations. When identifying such a generalization, the notion of classical negation plays a crucial role. We study two languages, $L_{1}$ and $L_{2}$, in both of which two negation signs are available: $\rightarrow$ and $\sim$. The latter is the usual GTS negation which transposes the players' roles, while the former will be interpreted via the notion of mode. Logic $L_{1}$ extends independence-friendly (IF) logic; $\rightarrow$ behaves as classical negation in $L_{1}$. Logic $L_{2}$ extends $L_{1}$, and it is shown to capture the $\Sigma_{1}^{2}$-fragment of third-order logic. Consequently the classical negation remains inexpressible in $L_{2}$.
\end{abstract}

\section{Introduction}

In game-theoretical semantics (GTS), which Hintikka originally formulated in [7], satisfaction conditions for formulas of first-order logic are formulated by associating a two-player zero-sum game $G(\varphi, \mathcal{M}, \gamma)$ between players $I$ and $I I$ with every formula $\varphi$, relevant model $\mathcal{M}$, and assignment $\gamma$ and defining $\varphi$ to be satisfied in $\mathcal{M}$ by $\gamma$ if there exists a winning strategy, w.s. for short, for player $I I$ in game $G(\varphi, \mathcal{M}, \gamma)$. The details of the definition of these semantic games can be phrased in a variety of ways, for example as follows.

We restrict attention to regular formulas, that is, formulas in which no two nested quantifiers carry the same variable and in which no variable appears both free and bound (for this terminology, see Caicedo, Dechesne, and Janssen [2]). Here and henceforth, if $\mathcal{M}$ is a model, $M$ is its domain. We denote the empty assignment by $\lambda$. Positions in semantic games are quadruples $(\psi, \mathcal{M}, \epsilon, \rho)$, where $\psi$ is a subformula of $\varphi ; \epsilon$ is an assignment; and $\rho$ is a bijective function of type $\{\mathbb{V}, \mathbb{F}\} \rightarrow\{I, I I\}$, termed a role distribution. Here $\mathbb{V}$ (verifier) and $\mathbb{F}$ (falsifier) are two roles. Henceforth

Received June 8, 2012; accepted January 10, 2013

2010 Mathematics Subject Classification: Primary 03B60, 03C80; Secondary 03B15

Keywords: game-theoretical semantics, higher-order logic, independence-friendly logic, negation

(C) 2014 by University of Notre Dame $\quad 10.1215 / 00294527-2798709$ 
we write $\rho_{0}$ for the role distribution satisfying $\rho_{0}(\mathbb{V})=I I$ and $\rho_{0}(\mathbb{F})=I$. The initial position of game $G(\varphi, \mathcal{M}, \gamma)$ is $\left(\varphi, \mathcal{M}, \gamma, \rho_{0}\right)$. Suppose, then, that position $(\psi, \mathcal{M}, \epsilon, \rho)$ has been reached. If $\circ \in\{\vee, \wedge\}$ and $\psi=\left(\theta_{1} \circ \theta_{2}\right)$, the next position is $\left(\theta_{i}, \mathcal{M}, \lambda, \rho\right)$. The term $\theta_{i}$ is chosen by player $\rho(\mathbb{V})$ if $\circ=\vee$ and by player $\rho(\mathbb{F})$ if $\circ=\wedge$. If $Q \in\{\exists, \forall\}$ and $\psi=Q x \theta$, the next position is $(\theta, \mathcal{M}, x \mapsto b, \rho)$, where $b \in M$ is chosen by $\rho(\mathbb{V})$ if $\mathrm{Q}=\exists$ and by $\rho(\mathbb{F})$ if $\mathrm{Q}=\forall$. If $\psi=\sim \theta$, the next position is $\left(\theta, \mathcal{M}, \lambda, \rho^{*}\right)$, where $\rho^{*}$ is the transposition of $\rho$, that is, a map satisfying $\rho^{*}(\mathbb{V})=\rho(\mathbb{F})$ and $\rho^{*}(\mathbb{F})=\rho(\mathbb{V})$. If $\psi$ is atomic, there are no further moves. If the sequence of positions from the initial position to the current position is $\left(\psi_{0}, \mathcal{M}, \epsilon_{0}, \rho_{0}\right), \ldots,\left(\psi_{n}, \mathcal{M}, \epsilon_{n}, \rho_{n}\right)$, this sequence induces an assignment $\gamma$ via the components $\epsilon_{i} \neq \lambda$. If $\psi_{n}$ is atomic and $\mathcal{M}, \gamma \models \psi_{n}$, then $\rho(\mathbb{V})$ wins, else $\rho(\mathbb{F})$ wins. ${ }^{1}$ A strategy of player $j \in\{I, I I\}$ is a tuple of strategy functions, one for each operator token for which the player must make a move. A strategy function yields a move for the corresponding operator token, depending on the adversary's earlier moves. It can be shown, if the axiom of choice is assumed, that a first-order formula $\varphi$ is satisfied in a structure $(\mathcal{M}, \gamma)$ according to the standard Tarskian semantics if and only if there exists a w.s. for player II in $G(\varphi, \mathcal{M}, \gamma)$ (see Hodges [14, p. 94]).

GTS is useful in connection with certain extensions of first-order logic. While strategy functions of a given player in first-order semantic games may take as arguments any earlier moves of the adversary, in independence-friendly logic (IF logic, here denoted $L_{\mathrm{IF}}$ ) there is a syntactic mechanism available for indicating that for specified operator tokens only some of the adversary's earlier moves are available as arguments (for $L_{\mathrm{IF}}$, see Hintikka [8]). The game rules for IF-logical games are the same as those for first-order games; the difference lies at the level of strategies. For example, if $\mathcal{M}$ is a model the domain of which consists of numbers 1 and 2 and $\varphi_{0}:=\forall x(\exists y /\{\forall x\}) x=y$, there are 4 plays in game $G\left(\varphi_{0}, \mathcal{M}\right)$, namely, $(1,1)$, $(1,2),(2,1)$, and $(2,2)$. The strategy function of player $I I$ for $(\exists y /\{\forall x\})$ must not take for its argument the value player $I$ has chosen for variable $x$; it must be a constant. Since choosing neither 1 nor 2 as a value for $y$ leads to a win for both possible values of $x$, there is no w.s. for $I I$ in game $G\left(\sim \varphi_{0}, \mathcal{M}\right)$, that is, $\varphi_{0}$ is not true in $\mathcal{M}$. On the other hand, neither is $\sim \varphi_{0}$ true in $\mathcal{M}$. In order for there to exist a w.s. for $I I$ in this game, it should be possible for $I I$, in the role of falsifier, to choose a value $a$ of $x$ so that for all values $b$ of $y$ that $I$ can choose, we have $a \neq b$. Since neither $\varphi$ nor $\sim \varphi$ is true in $\mathcal{M}$, the negation $\sim$ interpreted via the idea of switching roles does not capture classical negation $\neg$ : $\neg \psi$ is true precisely in those situations in which $\psi$ is not true. We will refer to $\sim$ as dual negation and $\neg$ as classical negation. We denote first-order logic formulated using $\sim$ as its negation symbol by $L_{\mathrm{FO}}$, while first-order logic with $\neg$ as its negation symbol is denoted by FO.

On various occasions Hintikka [8], [9], [10] has claimed that there cannot be a game rule for classical negation. Since satisfaction conditions formulated in terms of semantic games, as defined by Hintikka, are always expressible in the $\Sigma_{1}^{1}$-fragment of second-order logic (SO), it is indeed impossible that semantic games hence formulated could incorporate a rule for classical negation. ( $\Sigma_{1}^{1}$ is not closed under complementation.) We wish to show, however, that a game-theoretical analysis of classical negation is possible. This is achieved by enriching the structure of game positions by an additional component — to be termed a mode — and suitably interpreting the effect of a mode on the strategy level. This approach for capturing classical negation has 
its limits; it applies only to certain languages (including $L_{\mathrm{IF}}$ ). These limits will be identified. Related earlier research is discussed in Section 9.

In higher-order logics, we write $\equiv$ for logical equivalence and we use $\neg$ to denote negation. We write $L \leq L^{\prime}$ to indicate that logic $L$ can be translated into logic $L^{\prime}$; that is, for every $\varphi \in L$ there is $\psi_{\varphi} \in L^{\prime}$ such that $\mathcal{M}, \gamma \models \varphi$ if and only if $\mathcal{M}, \gamma \models \psi_{\varphi}$ for all suitable models $\mathcal{M}$ and assignments $\gamma$. Writing $L<L^{\prime}$ means that $L \leq L^{\prime}$ but $L^{\prime} \not \leq L$, while $L=L^{\prime}$ means that $L \leq L^{\prime}$ and $L^{\prime} \leq L$. We denote the empty tuple by ( ). When it serves clarity, we use angle brackets to mark a tuple: $\langle a, b, c\rangle$ means $(a, b, c)$. If $X$ is a set, we write $|X|$ for its cardinality. If $\mathcal{M}$ is a model of vocabulary $\tau$ with $P \in \tau$, then $P^{\mathcal{M}}$ stands for the interpretation of $P$ in $\mathcal{M}$. We abbreviate $\left(\sim \theta_{1} \vee \theta_{2}\right)$ by $\left(\theta_{1} \rightarrow \theta_{2}\right)$ and $\left(\left(\theta_{1} \rightarrow \theta_{2}\right) \wedge\left(\theta_{2} \rightarrow \theta_{1}\right)\right)$ by $\left(\theta_{1} \leftrightarrow \theta_{2}\right)$. Further, we write $\left(\theta_{1} \supset \theta_{2}\right)$ for $\left(\neg \theta_{1} \vee \theta_{2}\right)$ and $\left(\theta_{1} \subset \supset \theta_{2}\right)$ for $\left(\left(\theta_{1} \supset \theta_{2}\right) \wedge\left(\theta_{2} \supset \theta_{1}\right)\right)$. If $f$ is a function of type $A \rightarrow B$ and $C \subseteq A$, we write $\left.f\right|_{C}$ for the restriction of $f$ to the set $C$. For later use, we define the notion of game as follows.

Definition 1.1 (Game, play) A game between players $I$ and $I I$ is a quintuple $\left(X, S, p_{0}, c, u\right)$, the components of which are as follows. $X$ is a set and $p_{0} \in X$. The elements of $X$ are positions; $p_{0}$ is the initial position. Further, $S$ is function of type $X \rightarrow \mathcal{P}(X)$. If $p \in X$, the set $S(p)$ is said to consist of the possible successors of $p$. If the set $S(p)$ is empty, the position $p$ is terminal. The component $c$, called a player function, is defined on a subset of all nonterminal positions; its codomain is the set $\{I, I I\}$. If $c$ is defined on $p$, the elements of $S(p)$ are considered to be resulting from different moves available to player $c(p)$ at $p$. A play is any sequence $\left(p_{0}, \ldots, p_{n}\right)$ such that $p_{n}$ is terminal and $p_{i+1} \in S\left(p_{i}\right)$ for all $0 \leq i<n$. Any nonempty initial segment of a play is a partial play. Finally, $u$ is a utility function assigning to each play exactly one of the tuples (win, loss) or (loss, win). If $u(\pi)=($ win, loss $)$, we say that $I$ wins and $I I$ loses the play $\pi$, and if $u(\pi)=($ loss, win) we say that $I$ loses and $I I$ wins $\pi$.

\section{First-Order Semantic Games Relativized to Modes}

The existence of a w.s. for a given player is a strategic property of a game, but there are other properties of interest. Insofar as it makes sense to reason in terms of the class of strategies of a given player in the first place, we may pose questions pertaining to all those strategies, as well as to the existence of a strategy with such-and-such features. In particular, we may turn attention to the following property: for all strategies $g$ of player $I$ in $G(\varphi, \mathcal{M}, \gamma)$, there is a sequence of moves $\vec{b}$ of player $I I$ such that $I I$ wins the play determined by $g$ and $\vec{b}$. We proceed to study a greater variety of strategic properties than has been commonplace in connection with semantic games.

We will utilize semantic games whose positions are quintuples (rather than quadruples as in first-order semantic games), consisting not only of a formula, a model, an assignment, and a role distribution, but also a mode. We consider two modes, to be labeled as + and - . On the play level the modes have a very modest role, but at the strategic level they are of importance. The situation may be compared with the case of $L_{\mathrm{IF}}$, where the independence indications have no effect whatsoever at the play level, but they impose a constraint on strategies available to a given player. The modes will be used for game-theoretically interpreting a unary connective denoted by $\rightarrow$ and considered as a negation symbol, to be termed mode negation. This 
connective, hence interpreted, will capture classical negation in connection with certain logics.

Let us write $L_{0}$ for the fragment of $L_{\mathrm{FO}}$ consisting of formulas of the form $P_{1} \cdots P_{n} \chi$, where $1 \leq n<\omega$ and $\chi$ is a quantifier-free formula of a relational vocabulary and every $P_{i}$ is one of the symbols $\sim, \forall x_{i}$, and $\exists x_{i}$. The string $P_{1} \cdots P_{n}$ is the prefix and $\chi$ the matrix of the formula $P_{1} \cdots P_{n} \chi$. Note that if $P_{i}$ is a quantifier, the variable it carries is $x_{i}$. This syntactic restriction could be relaxed, but we stay with it for simplicity. So $\forall x_{1} \sim \exists x_{3} R\left(x_{1}, x_{3}\right)$ is a formula, but $\forall x_{1} \sim \exists x_{2} R\left(x_{1}, x_{2}\right)$ is not. By definition a (winning) strategy of player II in the semantic game $G(\varphi, \mathcal{M}, \gamma,+)$ with $\varphi \in L_{0}$ is simply any (winning) strategy for $I I$ in semantic game $G(\varphi, \mathcal{M}, \gamma)$, and a (winning) strategy of player $I$ in the semantic game $G(\varphi, \mathcal{M}, \gamma,-)$ is any (winning) strategy for $I$ in semantic game $G(\varphi, \mathcal{M}, \gamma)$. By contrast, a (winning) strategy of $I I$ in $G(\varphi, \mathcal{M}, \gamma,-)$ is any functional $F$ which for every strategy $f$ of $I$ in semantic game $G(\varphi, \mathcal{M}, \gamma)$ yields a sequence of moves $F(f)$ by $I I$ complying with the game rules of $G(\varphi, \mathcal{M}, \gamma)$ such that $f$ and $F(f)$ together determine a play of that game (won by $I I$ ); and a (winning) strategy of $I$ in $G(\varphi, \mathcal{M}, \gamma,+$ ) is any functional $F$ which for every strategy $f$ of $I I$ in $G(\varphi, \mathcal{M}, \gamma)$ yields a sequence of moves $F(f)$ by $I$ complying with the rules of $G(\varphi, \mathcal{M}, \gamma)$ such that $f$ and $F(f)$ together determine a play of that game (won by $I$ ). For $L_{0}$, we define semantic games relativized to a role distribution as follows: $G\left(\varphi, \mathcal{M}, \gamma, \rho_{0}, \star\right)$ equals $G(\varphi, \mathcal{M}, \gamma, \star)$, and $G\left(\varphi, \mathcal{M}, \gamma, \rho_{0}^{*}, \star\right)$ equals $G(\sim \varphi, \mathcal{M}, \gamma, \star)$.

Mode-relative semantic games for $L_{0}$ are mode-invariant; the proof uses the wellknown property of determinacy of standard semantic games for $L_{0}$. Mode invariance fails for more general languages to be studied in this paper.

Fact 2.1 (Mode invariance for $L_{0}$ ) $\quad$ For any $L_{0}$-formula $\varphi$, model $\mathcal{M}$, assignment $\gamma$, and player $j \in\{I, I I\}$, there is a w.s. for $j$ in game $G(\varphi, \mathcal{M}, \gamma,+)$ if and only if there is a w.s. for $j$ in game $G(\varphi, \mathcal{M}, \gamma,-)$.

Proof We begin with the case $j:=I I$. Suppose that there is a w.s., call it $g$, for $I I$ in $G(\varphi, \mathcal{M}, \gamma,+)$, but still there is no w.s. for $I I$ in $G(\varphi, \mathcal{M}, \gamma,-)$. Hence there is a strategy of player $I$ in $G(\varphi, \mathcal{M}, \gamma)$, call it $f$, such that for any sequence of moves by $I I$, it is $I$ who wins the resulting play. So the play obtained when $I I$ applies $g$ and $I$ applies $f$ is won by both players, which is impossible. Conversely, suppose that there is a w.s., call it $F$, for $I I$ in $G(\varphi, \mathcal{M}, \gamma,-)$. Suppose for contradiction that there is no w.s. for player $I I$ in $G(\varphi, \mathcal{M}, \gamma,+)$. By determinacy of semantic games for $L_{0}$, there is a w.s., call it $h$, for $I$ in $G(\varphi, \mathcal{M}, \gamma)$. Hence in $G(\varphi, \mathcal{M}, \gamma)$ the play determined by the strategy $h$ and the sequence of moves $F(h)$ is won by both players, which again is impossible. We may reason similarly if $j:=I$.

\section{Languages $L_{1}$ and $L_{2}$}

We begin to investigate to which extent and how we can game-theoretically capture classical negation in connection with certain extensions of $L_{0}$. In addition to $L_{0}$ with $\sim$ as its negation sign, we define two further languages: $L_{1}$ and $L_{2}$. Their formulas will have the general form $P_{1} \cdots P_{n} \chi$, where $0 \leq n<\omega$ and $\chi$ is a quantifier-free $L_{0}$-formula, every $P_{i}$ being one of the symbols $\sim, \neg,\left(\forall x_{i} / W_{i}\right)$, and $\left(\exists x_{i} / W_{i}\right)$, given that $W_{i}$ stands for a set of quantifiers $Q_{k} x_{k}$ with $1 \leq k<i$ and $\mathrm{Q}_{k} \in\{\forall, \exists\}$. The expressions $\left(\forall x_{i} / W_{i}\right)$ and $\left(\exists x_{i} / W_{i}\right)$ are called quantifiers. If clarity so demands, they may be termed slashed quantifiers in contradistinction to 
the expressions $\forall x_{i}$ and $\exists x_{i}$, which may then be referred to as plain quantifiers. Note that the expressions $W_{i}$ stand for sets of plain quantifiers, not slashed quantifiers. If $\left(\mathrm{Q} x_{i} / W_{i}\right)$ is a slashed quantifier, $/ W_{i}$ is its independence indication. If the set $W_{i}$ is empty, we adopt the convention of writing $\exists x_{i}$ for $\left(\exists x_{i} / W_{i}\right)$ and $\forall x_{i}$ for $\left(\forall x_{i} / W_{i}\right)$.

The polarity of expression $P_{i}$ in the prefix $P_{1} \cdots P_{n}$ is positive if the total number of negation signs in the string $P_{1} \cdots P_{i-1}$ is even (occurrences of both $\rightarrow$ and $\sim$ counted), otherwise it is negative. The quantifiers appearing in prefixes of formulas of the logics $L_{1}$ and $L_{2}$ are required to meet the following conditions.

- $L_{2}$ : Suppose $P_{i}=\left(\mathrm{Q}_{i} x_{i} / W_{i}\right)$ and $P_{j}=\left(\mathrm{Q}_{j} x_{j} / W_{j}\right)$ and $\mathrm{Q}_{i} x_{i} \in W_{j}$ with $i<j$. If $P_{i}$ and $P_{j}$ have the same polarity and $P_{i}$ is an existential (universal) quantifier, then $P_{j}$ is a universal (existential) quantifier. If, again, $P_{i}$ and $P_{j}$ have different polarities and $P_{i}$ is an existential (universal) quantifier, then also $P_{j}$ is an existential (universal) quantifier.

- $L_{1}$ : We have the condition for $L_{2}$ with the following additional requirement: if $P_{i}=\left(\mathrm{Q}_{i} x_{i} / W_{i}\right)$ and $P_{j}=\left(\mathrm{Q}_{j} x_{j} / W_{j}\right)$ and $\mathrm{Q}_{i} x_{i} \in W_{j}$ with $i<j$, then between $P_{i}$ and $P_{j}$ no $\rightarrow$-sign occurs in the prefix.

For example, if $\chi$ and $\theta$ are quantifier-free, then

$$
\rightarrow \forall x_{2} \exists x_{3} \forall x_{4}\left(\exists x_{5} /\left\{\forall x_{2}\right\}\right) \rightarrow \forall x_{7} \exists x_{8} \forall x_{9}\left(\exists x_{10} /\left\{\forall x_{7}\right\}\right) \chi
$$

is a formula of $L_{1}$ (but not of $L_{0}$ ), and

$$
\rightarrow \forall x_{2} \exists x_{3} \forall x_{4}\left(\exists x_{5} /\left\{\forall x_{2}\right\}\right) \rightarrow \forall x_{7} \exists x_{8} \forall x_{9}\left(\exists x_{10} /\left\{\forall x_{7}, \exists x_{3}, \exists x_{5}\right\}\right) \theta
$$

is a formula of $L_{2}$ (but not of $L_{1}$ ). Directly by definition, the sets of formulas of the logics introduced thus far are related as follows: $L_{0} \subsetneq L_{1} \subsetneq L_{2}$.

If $P_{1} \cdots P_{n} \chi$ is an $L_{2}$-formula, any string $P_{i} \cdots P_{n} \chi$ with $1 \leq i \leq n+1$ is its subformula. If $P_{i} \cdots P_{n} \chi$ is a subformula, the set Free $_{1}(\psi)$ of its free atomic variables consists of those variables $x_{j}$ appearing in $\chi$ for which there is no quantifier $\left(Q_{j} x_{j} / W_{j}\right)$ in the string $P_{i} \cdots P_{n}$. The set $\operatorname{Free}_{2}(\psi)$ of its free independence variables consists of variables $x_{j}$ such that there is a quantifier $\left(\mathrm{Q}_{k} x_{k} / W_{k}\right)$ in the string $P_{i} \cdots P_{n}$ and a quantifier $\mathrm{Q}_{j} x_{j}$ in $W_{k}$ with $j<i$. For example, if $\psi:=\left(\exists x_{7} /\left\{\forall x_{3}\right\}\right) R\left(x_{3}, x_{5}, x_{7}\right)$, then Free $_{1}(\psi)=\left\{x_{3}, x_{5}\right\}$ and Free $_{2}(\psi)=\left\{x_{3}\right\}$. Note that $\operatorname{Free}_{2}(\varphi)=\varnothing$ for all $\varphi \in L_{2}$. Subformulas containing free independence variables are not $L_{2}$-formulas.

The fragment of $L_{1}$ without $\rightarrow$ coincides, syntactically, with the fragment $L_{\mathrm{IF}}^{p r}$ of $L_{\mathrm{IF}}$ consisting of formulas with a prefix of slashed quantifiers followed by a quantifier-free matrix formula. By Theorem 7.2, the negation $\rightarrow$ actually behaves as classical negation in $L_{1}$. In fact, then, formulas $\rightarrow \theta$ with $\theta \in L_{\mathrm{IF}}^{p r}$ belong to what Hintikka [8] has called extended IF logic. The whole logic $L_{1}$ consists of formulas in prenex form of the fully extended IF logic (to be denoted $L_{\mathrm{FeIF}}$ ) discussed in [10], whereas $L_{2}$ goes even beyond $L_{\mathrm{FeIF}}$.

\section{Semantic Games Generalized}

We associate a semantics with $L_{2}$-formulas via a two-fold procedure. First, we define correlated semantic games; then we explain how the semantic attributes of interest are defined with reference to these games by using what we call metagames. Both semantic games and metagames are games in the sense of Definition 1.1. We define these games so that it will be absolutely clear what their corresponding components are (set $X$ of positions, successor function $S$, initial position $p_{0}$, player function $c$, 
utility function $u$ ), although we do not explicitly phrase the definitions as definitions of these five components.

Define a function $(\cdot)^{\prime}:\{+,-\} \rightarrow\{+,-\}$ by setting ${+^{\prime}}^{\prime}=-$ and $-^{\prime}=+$. A simple assignment of variable $x_{i}$ to a value $\xi$, denoted $x \mapsto \xi$, is the map $\left\{\left(x_{i}, \xi\right)\right\}$. Recall that $\lambda$ stands for the empty assignment. For every $L_{2}$-formula $\varphi=P_{1} \cdots P_{n} \chi$, suitable model $\mathcal{M}$, assignment $\gamma:$ Free $_{1}(\varphi) \rightarrow M$, and mode $\star \in\{+,-\}$, we associate a semantic game $G(\varphi, \mathcal{M}, \gamma, \star)$ defined as follows.

1. The initial position is $\left(\varphi, \mathcal{M}, \gamma, \rho_{0}, \star\right)$.

2. If $(\sim \psi, \mathcal{M}, \epsilon, \rho, \star)$ is a position, so is $\left(\psi, \mathcal{M}, \lambda, \rho^{*}, \star\right)$.

3. If $(\neg \psi, \mathcal{M}, \epsilon, \rho, \star)$ is a position, so is $\left(\psi, \mathcal{M}, \lambda, \rho^{*}, \star^{\prime}\right)$.

4. If $\left(\left(\mathrm{Q} x_{i} / W_{i}\right) \psi, \mathcal{M}, \epsilon, \rho, \star\right)$ is a position with $\mathrm{Q} \in\{\forall, \exists\}$, and $b \in M$, then $\left(\psi, \mathcal{M}, x_{i} \mapsto b, \rho, \star\right)$ is a position. If $\mathrm{Q}=\forall$, player $\rho(\mathbb{F})$ chooses one such position, else it is player $\rho(\mathbb{V})$ who chooses one such position.

5. If $(\psi, \mathcal{M}, \epsilon, \rho, \star)$ is a position, $\psi$ equals the matrix $\chi$, and the play which led to this position is $\pi=\left(\left(\psi_{0}, \mathcal{M}, \epsilon_{0}, \rho_{0}, \star_{0}\right), \ldots,\left(\psi_{n}, \mathcal{M}, \epsilon_{n}, \rho_{n}, \star_{n}\right)\right)$, then player $\rho(\mathbb{V})$ wins the play $\pi$ if $\mathcal{M}, \epsilon_{0}, \ldots, \epsilon_{n} \models \psi$, else $\rho(\mathbb{F})$ wins.

For simplicity we let plays terminate with the matrix formula, although it may not be atomic. Given that the $\epsilon_{i}$ are pairwise distinct variable assignments, the notation $\mathcal{M}, \epsilon_{0}, \ldots, \epsilon_{n} \models \psi$ means that $\mathcal{M}, \delta \models \psi$ in the usual sense of first-order logic, with $\delta=\bigcup_{0 \leq i \leq n} \epsilon_{i}$. Note that in the above game rules, $\epsilon_{0}=\gamma$. Viewed as sets, each $\epsilon_{i}$ with $i>0$ is either empty or a singleton.

Semantic games can be relativized to a role distribution by stipulating that $G\left(\varphi, \mathcal{M}, \gamma, \rho_{0}, \star\right)$ equals $G(\varphi, \mathcal{M}, \gamma, \star)$ and $G\left(\varphi, \mathcal{M}, \gamma, \rho_{0}^{*}, \star\right)$ equals $G(\sim \varphi, \mathcal{M}$, $\gamma, \star)$. If $\varphi$ is a sentence, $G(\varphi, \mathcal{M}, \star)$ equals $G(\varphi, \mathcal{M}, \lambda, \star)$. Note that independence indications play no role in the game rules; they will become operative at the strategy level. While the dual negation $\sim$ acts exclusively on the role distribution (transposing it), the mode negation $\rightarrow$ acts also on the mode (changing it). The impact of the modes will become manifest at the strategic level.

Recall that in Section 2 it was explained, for formulas $\varphi$ of $L_{0}$, what counts as a winning strategy of a given player in a semantic game $G(\varphi, \mathcal{M}, \gamma, \star)$. If $\varphi \in L_{0}$, write $\mathcal{M}, \gamma \models \varphi$ to indicate that there is a w.s. for player $I I$ in game $G(\varphi, \mathcal{M}, \gamma)$. We may observe that when applied to $L_{0}$-formulas, the connective $\rightarrow$ captures the classical negation.

Fact 4.1 Let $\varphi \in L_{0}$. For all suitable structures $(\mathcal{M}, \gamma)$, there is a w.s. for player II in game $G(\neg \varphi, \mathcal{M}, \gamma,+)$ if and only if $\mathcal{M}, \gamma \not \neq \varphi$.

Proof $\mathcal{M}, \gamma \not \forall \varphi$ if and only if there is no w.s. for player $I I$ in $G(\varphi, \mathcal{M}, \gamma)$ if and only if for every strategy of $I I$ in $G\left(\varphi, \mathcal{M}, \gamma, \rho_{0},+\right)$ there is a sequence of moves of $I$ such that $I$ wins the resulting play if and only if ( $\dagger$ ) for every strategy of $I$ in $G\left(\varphi, \mathcal{M}, \gamma, \rho_{0}^{*},+\right)=G\left(\varphi, \mathcal{M}, \gamma, \rho_{0}^{*}\right)$ there is a sequence of moves of $I I$ such that $I I$ wins the resulting play if and only if there is a w.s. for $I I$ in $G\left(\varphi, \mathcal{M}, \gamma, \rho_{0}^{*},-\right)$ if and only if there is a w.s. for $I I$ in $G\left(\neg \varphi, \mathcal{M}, \gamma, \rho_{0},+\right)$. The equivalence $(\dagger)$ holds because in $L_{0}$, any strategy for $I I$ in $G(\theta, \mathcal{N}, \delta)$ is a strategy for $I$ in $G(\sim \theta, \mathcal{N}, \delta)$, and vice versa.

\section{Metagames}

The strategic impact of a mode change with formulas discussed thus far (formulas of $L_{0}$ prefixed by $\rightarrow$ ) has been that of effecting a switch between the attributes "there 
is a strategy for player $I I$ such that for all sequences of moves by player $I$..." and "for all strategies of player $I$ there is a sequence of moves by player $I I$..." It is to be expected, then, that nested occurrences of $\rightarrow$ give rise to rather complicated strategic properties. For instance, insofar as $\rightarrow$ is to capture classical negation, on model $\mathcal{M}$ the formula $\rightarrow \forall x_{2} \exists x_{3} \rightarrow \forall x_{4} \exists x_{5} R\left(x_{2}, x_{3}, x_{4}, x_{5}\right)$ should state that for any function $f_{x_{3}}: M \rightarrow M$ there is a value $a$ of $x_{2}$ and a function $g_{x_{5}}: M \rightarrow M$ such that for any value $b$ of $x_{4}$, we have: $\langle a, f(a), b, g(b)\rangle \in R^{\mathcal{M}}$. The function $g_{x_{5}}$ may in principle depend on the function $f_{x_{3}}$. Indeed, the sentence should state the existence of functionals $F_{x_{2}}: M^{M} \rightarrow M$ and $F_{x_{5}}: M^{M} \rightarrow M^{M}$ such that for any function $f_{x_{3}}: M \rightarrow M$ and for any value $b$ of $x_{4}$, we have $\left\langle F_{x_{2}}\left(f_{x_{3}}\right), f_{x_{3}}\left(F_{x_{2}}\left(f_{x_{3}}\right)\right), b, F_{x_{5}}\left(f_{x_{3}}\right)(b)\right\rangle \in R^{\mathcal{M}}$. How should the relevant attributes be specified in connection with $\operatorname{logics} L_{1}$ and $L_{2}$ ? We opt for explicating this by using metagames. In terms of such metagames $\Gamma(\varphi, \mathcal{M}, \gamma, \star)$, we may conveniently discuss strategic properties of the object games - the plain semantic games $G(\varphi, \mathcal{M}, \gamma, \star)$ with $\varphi \in L_{i}$ with $i:=0,1,2$.

For $L_{0}$-formulas such metagames are extremely simple: in metagame $\Gamma(\varphi, \mathcal{M}$, $\gamma,+)$, first player $I I$ picks out, for each quantifier in the prefix for which it is his or her turn to move, what would be a corresponding strategy function $f_{i}$ in semantic game $G(\varphi, \mathcal{M}, \gamma)$. Then player $I$ picks out, for each quantifier in the prefix for which it would be her or his turn to move in semantic game $G(\varphi, \mathcal{M}, \gamma)$, an element $a_{j}$ of $M$. Metagame $\Gamma(\varphi, \mathcal{M}, \gamma,-)$ is played similarly, the difference being that it player $I$ who picks out what would be strategy functions for him in semantic game $G(\varphi, \mathcal{M}, \gamma)$, whereafter player II selects what would be moves for her in semantic game $G(\varphi, \mathcal{M}, \gamma)$. In either case, if the choices are $\vec{f}$ and $\vec{a}$ and the play of the semantic game $G(\varphi, \mathcal{M}, \gamma)$ determined by $\vec{f}$ and $\vec{a}$ is won by player $I I$, then player $I I$ wins the play $(\vec{f}, \vec{a})$ of the metagame $\Gamma(\varphi, \mathcal{M}, \gamma, \star)$, else player $I$ wins the play. There is no need to resort to the idea of metagame when explicating the notions of strategy and winning strategy for mode-relative $L_{0}$-games; indeed these notions were introduced for $L_{0}$ already in Section 2. However, in order to formulate the semantics of $L_{2}$ we need a generalization, and such a generalization is conveniently devised at the level of metagames. In the general case of $L_{2}$-formulas, plays of the metagame are structured as follows:

(tuple of functions ; tuple of moves), (tuple of functions ; tuple of moves), $\ldots$, (tuple of functions ; tuple of moves).

There is a finite number of rounds (tuple of functions, tuple of moves), in each of which first a finite number of functions are chosen, one by one, by one of the players (these we call function moves) - whereafter a finite number of elements from the domain are chosen, one by one, by the other player (element moves). The players alternate in making moves in the sense that one of the players makes function moves in the rounds with odd order position, and the other player in rounds with even order position. Once metagames and the corresponding notions of strategy are defined, we will declare that "strategy of player $j$ in a semantic game $G(\varphi, \mathcal{M}, \gamma, \star)$ " means "strategy of player $j$ in a metagame $\Gamma(\varphi, \mathcal{M}, \gamma, \star)$."

We need some auxiliary notions in order to define the general concept of metagame. If $S_{1} \cdots S_{r}$ is a string, any string $S_{i} \cdots S_{k}$ with $1 \leq i \leq k \leq r$ is its substring. The length of a string $S_{1} \cdots S_{r}$ equals $r$. Any string not containing the symbol $\rightarrow$ is $\rightarrow$-free. A substring $S_{i} \cdots S_{k}$ of a string $S_{1} \cdots S_{r}$ is maximally $\rightarrow$-free 
if it is $\rightarrow$-free and there is no $\rightarrow$-free substring of $S_{1} \cdots S_{r}$ of length greater than $(k-i)+1$ having $S_{i} \cdots S_{k}$ as its substring. A quantifier $P_{l}$ in the prefix $P_{1} \cdots P_{n}$ has existential force if the quantifier $P_{l}$ is existential and its polarity is positive in the prefix $P_{1} \cdots P_{n}$, or $P_{l}$ is universal and its polarity is negative in the prefix $P_{1} \cdots P_{n}$. The notion of a quantifier having universal force can be defined dually. Observe that the notions of existential and universal force are purely syntactic. (This will no longer be the case for the notions of the weak and strong force to be defined in Section 6.) There are two types of objects that the players may choose in the course of a metagame: elements of the domain and what we will call local strategy functions. It will depend on the position which types of objects can be chosen. Some further notions are needed to facilitate formulating the game rules for metagames.

Definition 5.1 (Barrier, local visibility) Let $\varphi:=P_{1} \cdots P_{n} \chi$ be an $L_{2}$-formula. Suppose that $P_{i}$ is a quantifier. If the prefix $P_{1} \cdots P_{i}$ is not $\rightarrow$-free, the backward barrier of the quantifier $P_{i}$ is the operator $P_{j}$ with $j<i$ such that $P_{j}=7$ and the string $P_{j+1} \cdots P_{i}$ is $\rightarrow$-free. Similarly, if the suffix $P_{i} \cdots P_{n}$ is not $\rightarrow$-free, the forward barrier of $P_{i}$ is the operator $P_{j}$ with $j>i$ such that $P_{j}=\rightarrow$ and the string $P_{i} \cdots P_{j-1}$ is $\rightarrow$-free. That is, the backward (forward) barrier of $P_{i}$ is the $\rightarrow$-sign closest to $P_{i}$ on the left (right) if one exists. The operators close to $P_{i}$ are those in the string $P_{k} \cdots P_{l}$ with $1 \leq k \leq i \leq l \leq n$, given that $\left(P_{k-1}\right.$ is the backward barrier of $P_{i}$ or if none exists $\left.k=1\right)$ and $\left(P_{l+1}\right.$ is the forward barrier of $P_{i}$ or if none exists $l=n$ ). If $P_{j}$ is the backward barrier of $P_{i}$, the operators in the string $P_{1} \cdots P_{j-1}$ are far from $P_{i}$. If $P_{i}=\left(\mathrm{Q} x_{i} / W_{i}\right)$ has existential force in $\varphi$, a quantifier $P_{k}$ is said to be locally visible for $P_{i}$ provided that the following conditions are satisfied: $k<i ; P_{k}$ is close to $P_{i} ; P_{k}$ has universal force in $\varphi$; and $P_{k}=\left(\mathrm{Q}^{\prime} x_{k} / W_{k}\right)$ with $\mathrm{Q}^{\prime} x_{k} \notin W_{i}$. Derivatively, if $P_{i}$ is a quantifier with existential force in $\varphi, \pi=\left(p_{0}, \ldots, p_{m-1}\right)$ is a partial play of a semantic game correlated with $\varphi$, and the subformula component of the position $p_{i-1}$ is $P_{i} \cdots P_{n} \chi$, the assignment locally visible at $\pi$ equals $\gamma \cup \delta$, where $\gamma$ is the assignment in $p_{0}$ and $\delta$ is the union of the simple assignments $x_{j} \mapsto b_{j}$ in positions $p_{j}$ such that quantifier $P_{j}$ is locally visible for $P_{i}$. These simple assignments result from having assigned a value to quantifier $P_{j}$ at position $p_{j-1}$. The notions of locally visible quantifier (assignment) can be defined dually for quantifiers with universal force.

Definition 5.2 (Local strategy function, local strategy) Consider a semantic game $G(\varphi, \mathcal{M}, \gamma, \star)$ with $\varphi:=O_{1} \cdots O_{n} \chi$. If $O_{i}=\left(\mathrm{Q} x_{i} / W_{i}\right)$ and $O_{i}$ has existential force in $\varphi$, a local strategy function for $O_{i}$ is a function $f_{i}$ satisfying the following: whenever $\pi=\left(p_{0}, \ldots, p_{i-1}\right)$ is a partial play of game $G(\varphi, \mathcal{M}, \gamma, \star)$ and $\delta$ is the assignment locally visible at $\pi$, the function $f_{i}$ is defined on the map $\delta$ and for some $b \in M$ we have $f_{i}(\delta)=x_{i} \mapsto b$. That is, the local strategy function $f_{i}$ tells player $I I$ which element $b \in M$ to assign to the variable $x_{i}$; the choice is allowed to depend on the assignment locally visible at $\pi$ and on nothing else. If $O_{j} \cdots O_{k}$ is a maximally $\rightarrow$-free string in of the prefix $O_{1} \cdots O_{n}$, a local strategy for player $I I$ is a sequence of local strategy functions, one for each $O_{i}$ with $j \leq i \leq k$ having existential force in $\varphi$. The notions of local strategy function and local strategy for player $I$ can be defined dually.

For any local strategy function $f$ there are fixed variables $x_{i_{1}}, \ldots, x_{i_{n+1}}$ such that $f$ takes assignments $\left\{\left(x_{i_{1}}, a_{i_{1}}\right), \ldots,\left(x_{i_{n}}, a_{i_{n}}\right)\right\}$ as arguments and yields a simple assignment $\left\{\left(x_{i_{n+1}}, a_{i_{n+1}}\right)\right\}$ as its value. Without danger of confusion we may, then, 
view local strategy functions as functions mapping tuples of elements $\left(a_{i_{1}}, \ldots, a_{i_{n}}\right)$ to elements $a_{i_{n+1}}$.

In semantic games for $L_{0}$ and even for $L_{\mathrm{IF}}$ all strategies are local strategies. Local strategies encode strategic reasonings restricted to parts of plays during which the negation $\rightarrow$ is not encountered. We will now define the notion of metagame, to be used in explicating the notions of truth and falsity of $L_{2}$-sentences. For every formula $\varphi=P_{1} \cdots P_{n} \chi$ of $L_{2}$, suitable structure $(\mathcal{M}, \gamma)$, and mode $\star \in\{+,-\}$, we associate a metagame $\Gamma(\varphi, \mathcal{M}, \gamma, \star)$ as follows.

1. The initial position is $\left(\varphi, \mathcal{M}, \gamma, \rho_{0}, \star\right)$.

2. If $\left(\neg P_{i} \cdots P_{n} \chi, \mathcal{M}, \epsilon, \rho, \star\right)$ is a position, the next position is $\left(P_{i} \cdots P_{n} \chi\right.$, $\left.\mathcal{M}, \lambda, \rho^{*}, \star^{\prime}\right)$.

3a. If $\left(P_{i} \cdots P_{n} \chi, \mathcal{M}, \epsilon, \rho, \star\right)$ is a position and $P_{i} \cdots P_{k}$ with $i \leq k \leq n$ is a maximally $\rightarrow$-free substring of $P_{i} \cdots P_{n}$ containing exactly $r$ dual negation symbols, the next position is

$$
\left(\left\langle\left(P_{i_{1}}, \ldots, P_{i_{s}}\right),\left(P_{j_{1}}, \ldots, P_{j_{t}}\right)\right\rangle P_{k+1} \cdots P_{n} \chi, \mathcal{M}, \lambda, \rho_{r}, \star\right),
$$

where the $P_{i_{y}}$ (resp., the $P_{j_{z}}$ ) are those quantifiers in the string $P_{i} \cdots P_{k}$ that have existential (universal) force in $\varphi$ and $\rho_{r}=\rho$ if $r$ is even, whereas $\rho_{r}=\rho^{*}$ if $r$ is odd.

3b. If $\left(\langle(),()\rangle P_{k+1} \cdots P_{n} \chi, \mathcal{M}, \epsilon, \rho, \star\right)$ is a position, the next position is $\left(P_{k+1} \cdots P_{n} \chi, \mathcal{M}, \lambda, \rho, \star\right)$.

4a. If $\left(\left\langle\left(P_{i_{r}}, \ldots, P_{i_{s}}\right),\left(P_{j_{1}}, \ldots, P_{j_{t}}\right)\right\rangle \rightarrow P_{k+1} \cdots P_{n} \chi, \mathcal{M}, \epsilon, \rho,+\right)$ is a position and $r \leq s$, player $I I$ chooses a local strategy function $f_{i_{r}}$ for $P_{i_{r}}$. The next position is $\left(\left\langle\left(P_{i_{r+1}}, \ldots, P_{i_{s}}\right),\left(P_{j_{1}}, \ldots, P_{j_{t}}\right)\right\rangle \rightarrow P_{k+1} \cdots P_{n} \chi\right.$, $\left.\mathcal{M}, x_{i_{r}} \mapsto f_{i_{r}}, \rho,+\right)$.

4b. If $\left(\left\langle(),\left(P_{j_{r}}, \ldots, P_{j_{t}}\right)\right\rangle \rightarrow P_{k+1} \cdots P_{n} \chi, \mathcal{M}, \epsilon, \rho,+\right)$ is a position and $r \leq t$, player $I$ picks out an element $b_{i_{r}} \in M$. The next position is $\left(\left\langle(),\left(P_{j_{r+1}}, \ldots, P_{j_{t}}\right)\right\rangle \rightarrow P_{k+1} \cdots P_{n} \chi, \mathcal{M}, x_{i_{r}} \mapsto b_{i_{r}}, \rho,+\right)$.

4c. If $\left(\left\langle\left(P_{i_{1}}, \ldots, P_{i_{s}}\right),\left(P_{j_{r}}, \ldots, P_{j_{t}}\right)\right\rangle \rightarrow P_{k+1} \cdots P_{n} \chi, \mathcal{M}, \epsilon, \rho,-\right)$ is a position and $r \leq t$, player $I$ chooses a local strategy function $f_{i_{r}}$ for $P_{i_{r}}$. The next position is $\left(\left\langle\left(P_{i_{1}}, \ldots, P_{i_{s}}\right),\left(P_{j_{r+1}}, \ldots, P_{j_{t}}\right)\right\rangle \rightarrow P_{k+1} \cdots P_{n} \chi\right.$, $\left.\mathcal{M}, x_{i_{r}} \mapsto f_{i_{r}}, \rho,-\right)$.

4d. If $\left(\left\langle\left(P_{i_{r}}, \ldots, P_{i_{s}}\right),()\right\rangle \rightarrow P_{k+1} \cdots P_{n} \chi, \mathcal{M}, \epsilon, \rho,-\right)$ is a position and $r \leq s$, player $I I$ picks out an element $b_{i_{r}} \in M$. The next position is $\left(\left\langle\left(P_{i_{r+1}}, \ldots, P_{i_{s}}\right),()\right\rangle \rightarrow P_{k+1} \cdots P_{n} \chi, \mathcal{M}, x_{i_{r}} \mapsto b_{i_{r}}, \rho,-\right)$.

5. If $(\psi, \mathcal{M}, \epsilon, \rho, \star)$ is a position, $\psi$ equals the matrix $\chi$, and the play that led to this position is $\pi=\left(\left(\psi_{0}, \mathcal{M}, \epsilon_{0}, \rho_{0}, \star_{0}\right), \ldots,\left(\psi_{n}, \mathcal{M}, \epsilon_{n}, \rho_{n}, \star_{n}\right)\right)$, then player $\rho(\mathbb{V})$ wins the play $\pi$ if $\mathcal{M}, \epsilon_{0}, \ldots, \epsilon_{n} \models \psi$, else $\rho(\mathbb{F})$ wins.

Metagames relativized to a role distribution are defined by stipulating that $\Gamma(\varphi, \mathcal{M}$, $\left.\gamma, \rho_{0}, \star\right)$ equals $\Gamma(\varphi, \mathcal{M}, \gamma, \star)$ and $\Gamma\left(\varphi, \mathcal{M}, \gamma, \rho_{0}^{*}, \star\right)$ equals $\Gamma(\sim \varphi, \mathcal{M}, \gamma, \star)$. Further, $\Gamma(\varphi, \mathcal{M}, \star)$ equals $\Gamma(\varphi, \mathcal{M}, \lambda, \star)$. Note that in item (3a) either $k=n$ or else $P_{k+1}=$ $\rightarrow$. Note also that in noninitial metagame positions there may appear simple assignments $\epsilon_{i}$ whose values are functions. In item (5), the notation $\mathcal{M}, \epsilon_{0}, \ldots, \epsilon_{n} \models \psi$ means $\mathcal{M}, \delta \models \psi$, where $\delta:$ Free $_{1}(\psi) \rightarrow M$ is the assignment defined as follows: if $\epsilon_{j}=\left(x_{i_{j}} \mapsto b_{i_{j}}\right)$, then $\delta\left(x_{i_{j}}\right)=b_{i_{j}}$, while if $\epsilon_{j}=\left(x_{i_{j}} \mapsto f_{i_{j}}\right)$ and $\eta$ is the assignment locally visible at the partial play $\left(\left(\psi_{0}, \mathcal{M}, \epsilon_{0}, \rho_{0}, \star_{0}\right), \ldots\right.$, $\left.\left(\psi_{j-1}, \mathcal{M}, \epsilon_{j-1}, \rho_{j-1}, \star_{j-1}\right)\right)$, then $\delta\left(x_{i_{j}}\right)=f_{i_{j}}(\eta)$. 
Remark Rule (3a) serves to group quantifiers of the string $P_{i} \cdots P_{k-1}$ into two subgroups according to whether they are of existential or universal force, by introducing a pair of lists of quantifiers: $\left\langle\left(P_{i_{1}}, \ldots, P_{i_{s}}\right),\left(P_{j_{1}}, \ldots, P_{j_{t}}\right)\right\rangle$. Rules (4a)-(4d) explicate how such pairs of lists are processed, one quantifier at a time, until both lists are empty. Thereafter rule (3b) applies and we are back with a string of symbols without pairs of list indicators. Expressions such as $\left\langle\left(P_{i_{r}}, \ldots, P_{i_{s}}\right),\left(P_{j_{1}}, \ldots, P_{j_{t}}\right)\right\rangle \rightarrow P_{k+1} \cdots P_{n} \chi$ are not formulas according to the syntax of $L_{2}$. We employ such auxiliary expressions in order to facilitate the formulation of the game rules. Observe that no special rule is needed for dual negation in metagames, since rule ( $3 \mathrm{a}$ ) is so formulated that it takes into account the possible occurrences of dual negations when forming the two lists of quantifiers and when determining the role distribution $\rho_{r}$. Finally note that rules $(4 a)-(4 d)$ are formulated in terms of players and not in terms of their roles. That is, for example, according to rule (4a), it is player $I I$ who chooses a local strategy function for $P_{i_{r}}$, irrespective of the role she or he happens to occupy in the corresponding object game when making a move for the quantifier $P_{i_{r}}$.

\section{Strategic Properties of Object Games}

If $\varphi$ is a sentence of logic $L_{0}$, a strategy $\vec{f}$ is winning for player $I I$ in semantic game $G(\varphi, \mathcal{M})$ if and only if making the initial moves $\vec{f}$ in metagame $\Gamma(\varphi, \mathcal{M},+)$ yields a win to $I I$ against any sequence of elements thereafter chosen by player $I$. Similarly, $\vec{g}$ is a w.s. for $I$ in $G(\varphi, \mathcal{M})$ if and only if the initial moves $\vec{g}$ in $\Gamma(\varphi, \mathcal{M},-)$ yield a win to $I$ against any sequence of elements chosen by $I I$. This observation will guide our generalizations: for truth, we turn attention to the positive mode and player $I I$, for falsity to the negative mode and player $I$.

Generally the existence of a w.s. for player II in metagame $\Gamma(\varphi, \mathcal{M}, \gamma,+)$ corresponds to a certain rather complicated strategic property of the object game $G(\varphi, \mathcal{M}, \gamma,+)$. In connection with $L_{1}$ the property takes the form

(there is a local strategy $f_{1}$ chosen by player $I I$ such that for any tuple $\vec{x}_{1}$ of elements chosen by $I$ ) (there is a local strategy $f_{2}$ chosen by player $I$ such that for any tuple of elements $\vec{x}_{2}$ chosen by $\left.I I\right) \ldots$

Logic $L_{2}$ introduces a further complication: those functionals that are utilized as strategy functions of player II in the metagame may not be allowed to take for arguments certain earlier moves by player $I$.

Consider a semantic game $G(\varphi, \mathcal{M}, \gamma, \star)$, where $\varphi=P_{1} \cdots P_{n} \chi$ is an $L_{2}$-formula. Suppose that $P_{r}$ is a quantifier with existential force in the prefix $P_{1} \cdots P_{n}$. The existential force of $P_{r}$ is said to be weak in the semantic game $G(\varphi, \mathcal{M}, \gamma, \star)$ if the initial mode $\star$ equals + and the number of $\rightarrow$-signs in the substring $P_{1} \cdots P_{r-1}$ is odd, or the initial mode $\star$ equals - and the number of $\rightarrow$-signs in the substring $P_{1} \cdots P_{r-1}$ is even. Its existential force is strong otherwise, that is, if the initial mode $\star$ equals + and the number of $\rightarrow$-signs in the substring $P_{1} \cdots P_{r-1}$ is even, or the initial mode $\star$ equals - and the number of $\rightarrow$-signs in the substring $P_{1} \cdots P_{r-1}$ is odd. Dually, a quantifier $P_{r}$ is of strong (weak) universal force in $G(\varphi, \mathcal{M}, \gamma, \star)$ if it is of universal force and either the initial mode is positive and $P_{r}$ is preceded by an odd (even) number of $\rightarrow$-signs, or else the initial mode is negative and $P_{r}$ is preceded by an even (odd) number of $\rightarrow$-signs. It should be noted that the notions of weak and strong force are not purely syntactic; they are relative not only to a 
prefix but also to the initial mode. In metagames players make function moves for quantifiers which are in the corresponding object game of strong force and element moves for quantifiers which are of weak force in the corresponding object game. We introduce a couple of auxiliary notions and then formulate the definition of strategy function applicable in connection with metagames.

Definition 6.1 (Visibility) Let $\varphi:=P_{1} \cdots P_{n} \chi$ be an $L_{2}$-formula. Suppose first that $P_{i}$ is a quantifier of existential force, and let $P_{j} \cdots P_{l}$ be the string of operators close to $P_{i}(j \leq i \leq l)$. A quantifier $P_{k}$ is said to be visible for $P_{i}$ if it satisfies the following conditions: $P_{k}$ is far from $P_{i}$ (whence $k<j$ ); $P_{k}$ has universal force in $\varphi$; and $x_{k} \notin W_{i}$. If $P_{i}$ has strong force, no other quantifiers are visible for $P_{i}$. If, again, $P_{i}$ has weak force, also all those $P_{k}$ are visible for $P_{i}$ that are close to $P_{i}$ and have universal force relative to $\varphi$. Derivatively, if $P_{r}$ is a quantifier with existential force in $\varphi, \pi=\left(p_{0}, \ldots, p_{m-1}\right)$ is a partial play of a metagame correlated with $\varphi$ and the expression $\left\langle\left(P_{r}, \ldots, P_{i_{s}}\right),\left(P_{j_{1}}, \ldots, P_{j_{t}}\right)\right\rangle \psi$ appears in the position $p_{m-1}$, the assignment visible at $\pi$ equals $\gamma \cup \delta$, where $\gamma$ is the assignment in $p_{0}$ and $\delta$ is the union of those simple assignments $x_{j} \mapsto \xi_{j}$ appearing in positions $p_{j}$ such that quantifier $P_{j}$ is visible for $P_{r}$. If $P_{j}$ has strong universal force, the object $\xi_{j}$ is a local strategy function, whereas if $P_{j}$ has weak universal force, $\xi_{j}$ is an element of the domain. The notions of visible quantifier and visible assignment can be defined dually for quantifiers with universal force.

The notion of locally visible assignment—defined for semantic games—must not be confused with the notion of visible assignment-defined for metagames. Also the notions of locally visible quantifier and visible quantifier must be kept apart. In particular if $P_{i}$ has strong existential force, none of the quantifiers locally visible for $P_{i}$ are visible for $P_{i}$. Note also that if $P_{i}$ has weak existential force, all quantifiers close to it are visible for $P_{i}$-including those that lie in its syntactic scope. In accordance with the metagame rules, the adversary will have associated a local strategy function with all those quantifiers before an element is assigned to $P_{i}$.

Definition 6.2 (Strategy in a metagame) Let $\varphi=P_{1} \cdots P_{n} \chi$ be an $L_{2}$-formula. If $P_{i}$ is a quantifier of existential force, a strategy function of player $I I$ for $P_{i}$ in metagame $\Gamma(\varphi, \mathcal{M}, \gamma, \star)$ is a function $F_{i}$ satisfying the following. Whenever $\pi=$ $\left(p_{0}, \ldots, p_{i-1}\right)$ is a partial play of $\Gamma(\varphi, \mathcal{M}, \gamma, \star)$ and the assignment visible at $\pi$ is $\delta$, the function $F_{i_{r}}$ is defined on $\delta$ and we have the following:

- if $p_{i-1}=\left(\left\langle\left(P_{i_{r}}, \ldots, P_{i_{s}}\right),\left(P_{j_{1}}, \ldots, P_{j_{t}}\right)\right\rangle \psi, \mathcal{M}, \epsilon, \rho,+\right)$ and $P_{i_{r}}$ has strong force, then $F_{i_{r}}$ tells player $I I$ which local strategy function to pick; that is, there is a local strategy function $f_{i_{r}}$ for $P_{i_{r}}$ such that $F_{i_{r}}(\delta)=f_{i_{r}}$;

- if $\left(\left\langle\left(P_{i_{r}}, \ldots, P_{i_{s}}\right),()\right\rangle \psi, \mathcal{M}, \epsilon, \rho,-\right)$ and $P_{i_{r}}$ has weak force, then $F_{i_{r}}$ tells player $I I$ which element $b \in M$ to pick, that is, $F_{i_{r}}(\delta) \in M$.

A strategy $F$ for player $I I$ is a sequence of strategy functions, one strategy function $F_{i}$ for each quantifier $P_{i}$ of existential force in the prefix. We say that player $I I$ has used strategy $F$ in a play $\left(p_{0}, \ldots, p_{m}\right)$ of game $\Gamma(\varphi, \mathcal{M}, \gamma, \star)$ if the following two conditions are met for all $i \leq m$ :

- if $p_{i-1}=\left(\left\langle\left(P_{i_{r}}, \ldots, P_{i_{s}}\right),\left(\left\langle P_{j_{1}}, \ldots, P_{j_{t}}\right)\right\rangle \psi, \mathcal{M}, \epsilon, \rho,+\right)\right.$, and $P_{i_{r}}$ has strong force, and $\delta_{i-1}$ is the assignment visible to player $I I$ at $\left(p_{0}, \ldots, p_{i-1}\right)$, then $p_{i}=\left(\left\langle\left(P_{i_{r+1}}, \ldots, P_{i_{s}}\right),\left(P_{j_{1}}, \ldots, P_{j_{t}}\right)\right\rangle \psi, \mathcal{M}, x_{i_{r}} \mapsto F_{i_{r}}\left(\delta_{i-1}\right), \rho,+\right)$; 
- if $p_{i-1}=\left(\left\langle\left(P_{i_{r}}, \ldots, P_{i_{s}}\right),()\right\rangle \psi, \mathcal{M}, \epsilon, \rho,-\right)$, and $P_{i_{r}}$ has weak force, and $\delta_{i-1}$ is the assignment visible to player $I I$ at $\left(p_{0}, \ldots, p_{i-1}\right)$, then $p_{i}=\left(\left\langle\left(P_{i_{r+1}}, \ldots, P_{i_{s}}\right),()\right\rangle \psi, \mathcal{M}, x_{i_{r}} \mapsto F_{i_{r}}\left(\delta_{i-1}\right), \rho,-\right)$.

The notions of strategy and using a strategy can be defined dually for player $I$. A strategy is winning for player $j$ if for all sequences of moves by his or her adversary, it yields moves so that the resulting play is won by player $j$.

Similarly to the case of local strategy functions, in the case of strategy functions we may ignore the fact that their arguments are assignments $\left\{\left(x_{i_{1}}, \xi_{i_{1}}\right), \ldots,\left(x_{i_{n}}, \xi_{i_{n}}\right)\right\}$ and their values are simple assignments $\left\{\left(x_{i_{n+1}}, \xi_{i_{n+1}}\right)\right\}$; we may simply treat them as functions taking tuples $\left(\xi_{i_{1}}, \ldots, \xi_{i_{n}}\right)$ as their arguments and yielding the object $\xi_{i_{n+1}}$ as their value.

The effect of independence indications $/ W_{i}$ that results from the definitions of strategy and local strategy may be summarized as follows. If $P_{1} \cdots P_{n} \chi$ is a formula and $P_{i}=\left(\mathrm{Q}_{i} x_{i} / W_{i}\right)$ appears in the maximally $\rightarrow$-free string $P_{h} \cdots P_{k}$, let $\left(V_{i}, U_{i}\right)$ be a partition of $W_{i}$ such that quantifiers in $V_{i}$ occur in the string $P_{1} \cdots P_{h-1}$ (i.e., are far from $P_{i}$ ), while those in $U_{i}$ occur in the string $P_{h} \cdots P_{k}$ (i.e., are close to $P_{i}$ ). The set $V_{i}$ regulates strategy functions for $P_{i}$ in metagames but does not affect the play level, while the set $U_{i}$ regulates its local strategy functions and therefore affects the play level in metagames. If $P_{i}$ is of strong existential force, the quantifiers in $U_{i}$ determine the type of the local strategy functions for $P_{i}$. If, again, $P_{i}$ is of weak existential force, the quantifiers in $U_{i}$ play no role whatsoever. It is obvious they cannot have a role at the play level, since the move corresponding to $P_{i}$ is an element of the domain if $P_{i}$ is of weak force. However, we do not wish quantifiers in $U_{i}$ to have a role at the strategy level either. That is, if $P_{i}$ has weak existential force, $P_{j}$ is a quantifier of universal force close to $P_{i}$ (i.e., $h \leq j \leq k$ ), and $\mathrm{Q}_{j} x_{j} \in W_{i}$, we wish the indicated independence vis-à-vis $\mathrm{Q}_{j} x_{j}$ to be vacuous. We take this to be motivated by the behavior of $L_{\mathrm{IF}}$. Locally we are interested-in any given maximally $\rightarrow$-free string - in strategies of one player only, in analogy with the case of $L_{\mathrm{IF}}$, in connection with which, considering the truth (falsity) of a sentence, only independence indications of quantifiers with existential (universal) force matter, that is, quantifiers of only one type are interpreted in terms of strategies and restrictions imposed on them. As to the set $V_{i}$, it determines which earlier moves by the adversary are allowed as arguments of the strategy function for $P_{i}$. If $P_{i}$ has weak existential force, among those earlier moves there are automatically in particular all moves that the adversary has made for the quantifiers of strong universal force earlier in the current maximally $\rightarrow$-free string, among which there may well be quantifiers close to $P_{i}$ in the syntactic scope of $P_{i}$.

In order to have available the notion of (winning) strategy also in object games, we stipulate the following.

Definition 6.3 (Strategy in an object game) Let $\varphi$ be an $L_{2}$-formula, and let $j \in\{I, I I\}$. We say that a tuple of functions $F$ is a (winning) strategy for player $j$ in an object game $G(\varphi, \mathcal{M}, \gamma, \star)$ if $F$ is a (winning) strategy for $j$ in the metagame $\Gamma(\varphi, \mathcal{M}, \gamma, \star)$.

The semantic attributes we are interested in are defined as follows.

Definition 6.4 (Satisfaction, satisfaction equivalence) If $\varphi \in L_{2}, j \in\{I, I I\}$, and $\star \in\{+,-\}$, we write $\mathcal{M}, \gamma \models_{j}^{\star} \varphi$, if there exists a w.s. for player $j$ in game 
$G(\varphi, \mathcal{M}, \gamma, \star)$. If $\mathcal{M}, \gamma \models_{I I}^{+} \varphi$, we say that formula $\varphi$ is satisfied in model $\mathcal{M}$ by assignment $\gamma$. If $\varphi$ is a sentence satisfied in $\mathcal{M}$ by $\lambda$, we say that it is true in $\mathcal{M}$, symbolically $\mathcal{M} \models_{I I}^{+} \varphi$. If $\mathcal{M}, \gamma \models_{I}^{-} \varphi$, we speak of dissatisfaction and falsity. Formulas $\varphi\left(x_{1}, \ldots, x_{n}\right)$ and $\psi\left(x_{1}, \ldots, x_{n}\right)$ are satisfaction-equivalent if for all suitable structures $(\mathcal{M}, \gamma)$, we have $\mathcal{M}, \gamma \models_{I I}^{+} \varphi$ if and only if $\mathcal{M}, \gamma \models_{I I}^{+} \psi$. If $\varphi$ and $\psi$ are sentences, we speak of truth equivalence. Formulas $\varphi\left(x_{1}, \ldots, x_{n}\right)$ and $\psi\left(x_{1}, \ldots, x_{n}\right)$ are strongly equivalent if for all suitable $(\mathcal{M}, \gamma)$, all $j \in\{I, I I\}$, and all $\star \in\{+,-\}$, we have $\mathcal{M}, \gamma \models_{j}^{\star} \varphi$ if and only if $\mathcal{M}, \gamma \models_{j}^{\star} \psi$.

In Sections 7 and 8, we will study the expressivity of the logics $L_{1}$ and $L_{2}$. The notation $L \leq L^{\prime}$ used when comparing the expressive powers of logics $L$ and $L^{\prime}$ was introduced at the end of Section 1 supposing that the satisfaction relation of both $\operatorname{logics}$ is $\models$. When one of the logics is $L_{1}$ or $L_{2}$, it must be understood that the intended satisfaction relation for that logic is $\models_{I I}^{+}$.

The following interconnections of the relations just defined are immediate. ${ }^{2}$

Fact 6.5 Let $\varphi \in L_{2}$ be arbitrary. Then

(a) $\mathcal{M}, \gamma \models_{I}^{+} \varphi$ if and only if $\mathcal{M}, \gamma \models_{I I}^{-} \sim \varphi$ if and only if $\mathcal{M}, \gamma \models_{I I}^{+} \rightarrow \varphi$,

(b) $\mathcal{M}, \gamma \models_{I}^{-} \varphi$ if and only if $\mathcal{M}, \gamma \models_{I I}^{+} \sim \varphi$ if and only if $\mathcal{M}, \gamma \models_{I I}^{-} \rightarrow \varphi$.

We say that a formula $\varphi$ is bivalent if either $\mathcal{M}, \gamma \models_{I I}^{+} \varphi$ or $\mathcal{M}, \gamma \models_{I}^{-} \varphi$, for all suitable structures $(\mathcal{M}, \gamma)$.

Observation 6.6 Not all negated formulas $\rightarrow \varphi$ are bivalent. For example, let $\psi_{0}:=\forall x(\exists y /\{\forall x\}) x=y$, and let $\mathcal{M}_{0}$ be a model with at least two elements. Then $\psi_{0}$ is neither true nor false: neither $\mathcal{M}_{0} \forall_{I I}^{+} \psi_{0}$ nor $\mathcal{M}_{0} \not \forall_{I}^{-} \psi_{0}$. Yet $\rightarrow \longrightarrow \psi_{0}$ is strongly equivalent to $\psi_{0}$, so $\rightarrow \longrightarrow \psi_{0}$ is a formula prefixed by $\rightarrow$ which is not bivalent. In Theorem 7.2 we will see that for all $L_{1}$-sentences $\varphi$ and models $\mathcal{M}$, we have that $\rightarrow \varphi$ is true in $\mathcal{M}$ if and only if $\varphi$ is not true in $\mathcal{M}$. By what was just observed, the nontruth of $\rightarrow \varphi$ does not in general imply its falsity.

Whenever $P_{1} \cdots P_{n} \chi$ is an $L_{2}$-formula, let $P_{1}^{+} \ldots P_{n}^{+}$be the string defined as follows: $P_{i}^{+}=P_{i}$ if $P_{i}$ is not a quantifier; $P_{i}^{+}=\mathrm{Q}_{i} x_{i}$ if $P_{i}=\left(\mathrm{Q}_{i} x_{i} / W_{i}\right)$ is a quantifier of weak universal force; $P_{i}^{+}=\left(\mathrm{Q}_{i} x_{i} / U_{i}^{\prime}\right)$ if $P_{i}=\left(\mathrm{Q}_{i} x_{i} / V_{i} \cup U_{i}\right)$ is a quantifier of strong universal force and $U_{i}^{\prime}=\left\{\mathrm{Q}: \mathrm{Q} \in U_{i}\right.$ and $\mathrm{Q}$ is of existential force $\} ; P_{i}^{+}=\left(\mathrm{Q}_{i} x_{i} / V_{i}^{\prime}\right)$ if $P_{i}=\left(\mathrm{Q}_{i} x_{i} / V_{i} \cup U_{i}\right)$ is of weak existential force and $V_{i}^{\prime}=\left\{\mathrm{Q}: \mathrm{Q} \in V_{i}\right.$ and $\mathrm{Q}$ is of universal force $\}$; and $P_{i}^{+}=\left(\mathrm{Q}_{i} x_{i} / W_{i}^{\prime}\right)$ if $P_{i}=\left(\mathrm{Q}_{i} x_{i} / W_{i}\right)$ is of strong existential force and $W_{i}^{\prime}=\left\{\mathrm{Q}: \mathrm{Q} \in W_{i}\right.$ and $\mathrm{Q}$ is of universal force $\}$; here the partition $\left(V_{i}, U_{i}\right)$ of $W_{i}$ is defined as explained above. We define the string $P_{1}^{-} \ldots P_{n}^{-}$in a dual fashion. The following fact is a direct consequence of the semantics.

Fact 6.7 A formula $P_{1} \cdots P_{n} \chi$ of $L_{2}$ is satisfied (resp., dissatisfied) in $\mathcal{M}$ by $\gamma$ if and only if $P_{1}^{+} \ldots P_{n}^{+} \chi$ is satisfied (resp., $P_{1}^{-} \ldots P_{n}^{-} \chi$ is dissatisfied) in $\mathcal{M}$ by $\gamma$.

If we write $L_{1}^{\prime}$ for the fragment of logic $L_{1}$ whose formulas do not use the connective $\rightarrow$, then $L_{1}^{\prime}=\Sigma_{1}^{1}$. For, $L_{1}^{\prime}$ coincides not only syntactically but also semantically with $L_{\mathrm{IF}}^{p r}$, and by well-known results this fragment coincides with $\Sigma_{1}^{1}$ (see [8]). Before proceeding to further expressivity issues, let us consider some examples. Below, when no confusion is likely, we allow using in the syntax variables such as $x, y, z$, and so on, requiring, however, that the formulas be regular in the sense mentioned in the beginning of this paper. 
Example 6.8 (Logic $\left.L_{1}\right) \quad$ We claim that the $L_{1}$-sentence

$$
\forall t \rightarrow \forall x \forall y(\exists z /\{\forall y\})(\exists v /\{\forall x\}) \rightarrow([x=y \leftrightarrow z=v] \rightarrow t=z),
$$

call it $\varphi$, is true in a model $\mathcal{M}$ if and only if the domain $M$ is finite. Its truth condition can be expressed by the $\Pi_{1}^{1}$-sentence $\forall t \forall f \forall g \exists x \exists y([x=y \subset \supset$ $\mathrm{f}(x)=\mathrm{g}(y)] \supset t=\mathrm{f}(x))$. What this sentence states is that if $t \in M, f \in M^{M}$ and $g \in M^{M}$ are arbitrary, either $f \neq g$ (in which case there are $a, b \in M$ so that $a=b$ and $f(a) \neq g(b))$ or $f=g$ but $f$ is not injective (and so there are $a, b \in M$ such that $a \neq b$ and $f(a)=g(b))$ or else $f=g$ and $f$ is injective and there is $a \in M$ so that $t=f(a)$. That is, the $\Pi_{1}^{1}$-sentence states that every injective function of type $M \rightarrow M$ is surjective, that is, that the domain $M$ is finite. Let us check directly in terms of the semantics of $L_{1}$ that $\varphi$ expresses the finiteness of the domain.

First suppose that $M$ is finite. Define a strategy $\left(F_{\forall x}, F_{\forall y}\right)$ for player $I I$ in metagame $\Gamma(\varphi, \mathcal{M},+)$ as follows. If $a, f$, and $g$ are the choices of player $I$ for $\forall t$, $(\exists z /\{\forall y\})$, respectively, $(\exists v /\{\forall x\})$, let $F_{\forall x}(a, f, g)=F_{\forall y}(a, f, g)=b$ if $f \neq g$, where $b$ satisfies $f(b) \neq g(b)$; further let $F_{\forall x}(a, f, g)=b^{\prime}$ and $F_{\forall y}(a, f, g)=b^{\prime \prime}$ if $f=g$ but $f$ is not injective, where $b^{\prime}, b^{\prime \prime}$ with $b^{\prime} \neq b^{\prime \prime}$ satisfy $f\left(b^{\prime}\right)=g\left(b^{\prime \prime}\right)$; finally, if $f=g$ and $f$ is injective, choose the value of $F_{\forall y}(a, f, g)$ in an arbitrary fashion, and let $F_{\forall x}(a, f, g)=e$ for the uniquely determined $e$ such that $f(e)=a$. (Such a value $e$ exists because $M$ is finite.) Clearly the strategy $\left(F_{\forall x}, F_{\forall y}\right)$ is winning for $I I$. Note that the values of the strategy functions $F_{\forall x}$ and $F_{\forall y}$ depend on function choices of player $I$, and if $f=g$ and $f$ is injective, the value also depends on the element choice of player $I$. Second, suppose $\mathcal{M} \models_{I I}^{+} \varphi$, and let $\left(F_{\forall x}, F_{\forall y}\right)$ be a w.s. for $I I$ in metagame $\Gamma(\varphi, \mathcal{M},+)$. We show $M$ to be finite. Let $f: M \rightarrow M$ be injective; let $a \in M$ be arbitrary. We must show that there is $b \in M$ such that $a=f(b)$. Consider any play of the metagame where the choices of player $I$ are, in the order in which they are made, $a, f$, and $f$. Letting $b:=F_{\forall x}(a, f, f)$, we have $a=f(b)$, since $\left(F_{\forall x}, F_{\forall y}\right)$ is a winning strategy.

Example 6.9 (Logic $\left.L_{2}\right) \quad$ Let $\psi(A, B, C)$ be the $L_{2}$-sentence

$$
\begin{gathered}
\forall t \rightarrow \forall x \forall x^{\prime}\left(\exists y /\left\{\forall x^{\prime}\right\}\right)\left(\exists y^{\prime} /\{\forall x\}\right) \rightarrow\left(\exists v /\left\{\forall t, \exists y^{\prime}\right\}\right)\left(\exists v^{\prime} /\{\forall t, \exists y\}\right) \\
\left([ A ( t ) \wedge B ( y ) \wedge B ( y ^ { \prime } ) ] \rightarrow \left[x=x^{\prime} \wedge A(x) \wedge C(v) \wedge C\left(v^{\prime}\right)\right.\right. \\
\left.\left.\wedge\left(\left[y \neq y^{\prime} \wedge v \neq v^{\prime}\right] \vee\left[x=t \wedge y=y^{\prime} \wedge v=v^{\prime}\right]\right)\right]\right) .
\end{gathered}
$$

We claim that whenever $\mathcal{M}$ is a (finite or infinite) model with each of the sets $A^{\mathcal{M}}$, $B^{\mathcal{M}}, C^{\mathcal{M}}$ nonempty, we have $\mathcal{M} \models{ }_{I I}^{+} \psi(A, B, C)$ if and only if $\left|B^{\mathcal{M}}\right|^{\left|A^{\mathcal{M}}\right|} \leq\left|C^{\mathcal{M}}\right|$.

Left to right. Let $\left(F_{\forall x}, F_{\forall x^{\prime}}, F_{\exists v}, F_{\exists v^{\prime}}\right)$ be a w.s. for player II in metagame $\Gamma(\psi(A, B, C), \mathcal{M},+)$. Thus, there are functionals $S$ and $S^{\prime}$ of type $M^{M} \rightarrow C^{\mathcal{M}}$ with $S=F_{\exists v}$ and $S^{\prime}=F_{\exists v^{\prime}}$ such that for all maps $f$ and $f^{\prime}$ of type $M \rightarrow B^{\mathcal{M}}$, either there is an element $b=F_{\forall x}=F_{\forall x^{\prime}}$ in $A^{\mathcal{M}}$ such that $f(b) \neq f^{\prime}(b)$ and $S(f) \neq S^{\prime}\left(f^{\prime}\right)$, or else for all $c \in A^{\mathcal{M}}$ we have $f(c)=f^{\prime}(c)$ and $S(f)=S^{\prime}\left(f^{\prime}\right)$. Fix an element $d \in C^{\mathcal{M}}$, and for all $g: A^{\mathcal{M}} \rightarrow B^{\mathcal{M}}$, let $h_{g}$ be the map of type $M \rightarrow B^{\mathcal{M}}$ satisfying $h_{g}(a)=g(a)$ if $a \in A^{\mathcal{M}}$, and $h_{g}(a)=d$ otherwise. Define maps $T$ and $T^{\prime}$ of type $\left(B^{\mathcal{M}}\right)^{A^{\mathcal{M}}} \rightarrow C^{\mathcal{M}}$ by setting $T(g)=S\left(h_{g}\right)$ and $T^{\prime}(g)=S^{\prime}\left(h_{g}\right)$ for all maps $g: A^{\mathcal{M}} \rightarrow B^{\mathcal{M}}$. By the properties of $S$ and $S^{\prime}$, $T=T^{\prime}$ and this function is injective. 
Right to left. Suppose that there is an injective function $T:\left(B^{\mathcal{M}}\right)^{A^{\mathcal{M}}} \rightarrow C^{\mathcal{M}}$. Define a strategy $\left(F_{\forall x}, F_{\forall x^{\prime}}, F_{\exists v}, F_{\exists v^{\prime}}\right)$ for player $I I$ in metagame $\Gamma(\psi(A, B, C)$, $\mathcal{M},+)$ as follows. Suppose that $a \in M, f \in M^{M}$, and $f^{\prime} \in M^{M}$ are the choices of player $I$ for $\forall t,\left(\exists y /\left\{\forall x^{\prime}\right\}\right)$, respectively, $\left(\exists y^{\prime} /\{\forall x\}\right)$. Let $g=\left.f\right|_{A^{\mathcal{M}}}$ and $g^{\prime}=\left.f^{\prime}\right|_{A^{\mathcal{M}}}$. We may suppose that the images of these maps are contained in $B^{\mathcal{M}}$, for if they are not, there is $c \in M$ with $\left(g \cup g^{\prime}\right)(c) \notin B^{\mathcal{M}}$ such that by choosing $c$ as the common value of $x$ and $x^{\prime}$ player $I I$ wins the resulting play, no matter which moves are made for $\forall t, \exists v$, and $\exists v^{\prime}$. Likewise we may suppose $a \in A^{\mathcal{M}}$. Now, we put $F_{\exists v}=F_{\exists v^{\prime}}=S$, where $S: M^{B^{\mathcal{M}}} \rightarrow M$ is a functional defined as follows: $S(h)=T\left(\left.h\right|_{A^{\mathcal{M}}}\right)$, for all $h \in M^{B^{\mathcal{M}}}$. Further, (1) if $g=g^{\prime}$, we set $F_{\forall x}=F_{\forall x^{\prime}}=a$, while (2) if $g \neq g^{\prime}$, we put $F_{\forall x}=F_{\forall x^{\prime}}=b$, where $b$ is a fixed element satisfying $g(b) \neq g^{\prime}(b)$. Let us check that player $I I$ wins the resulting play in both cases. Consider case (1) first. The assignment $\delta$ induced by these moves satisfies $\delta(t)=\delta(x)=\delta\left(x^{\prime}\right)=a$; and $\delta(v)=S(f)=T(g)=T\left(g^{\prime}\right)=S\left(f^{\prime}\right)=\delta\left(v^{\prime}\right)$; and $\delta(y)=f(a)=g(a)=g^{\prime}(a)=\delta\left(y^{\prime}\right)$ (because $a \in A^{\mathcal{M}}$ ). Hence in particular $\mathcal{M}, \delta \models C(v) \wedge C\left(v^{\prime}\right) \wedge x=t \wedge y=y^{\prime} \wedge v=v^{\prime}$. In case (2), the induced assignment $\delta$ satisfies the following: $\delta(x)=\delta\left(x^{\prime}\right)=b$; and $\delta(v)=S(f)=T(g) \neq T\left(g^{\prime}\right)=S\left(f^{\prime}\right)$ (since $T$ is injective); and $\delta(y)=f(b)=g(b) \neq g^{\prime}(b)=\delta\left(y^{\prime}\right)$ (because $b \in A^{\mathcal{M}}$ ). Hence in particu$\operatorname{lar} \mathcal{M}, \delta \models C(v) \wedge C\left(v^{\prime}\right) \wedge A(x) \wedge y \neq y^{\prime} \wedge v \neq v^{\prime}$.

Below we will use the following fact.

Fact 6.10 Every formula $\varphi$ of $L_{2}$ is strongly equivalent to a formula of $L_{2}$ whose prefix contains no $\sim$-sign.

Proof Let us first agree on the following notation. Suppose that $W$ is a set of plain quantifiers. If $\mathrm{Q} x \in W$, write $W_{\mathrm{Q} x}^{d}$ for the result of replacing $\mathrm{Q} x$ in $W$ by its dual; otherwise let $W_{\mathrm{Q} x}^{d}=W$. (The dual of $\exists x$ is $\forall x$, and vice versa.) If $\left(\mathrm{Q}^{\prime} x^{\prime} / W\right)$ is a slashed quantifier, $\left(\mathrm{Q}^{\prime} x^{\prime} / W\right)_{\mathrm{Q} x}^{d}$ denotes $\left(\mathrm{Q}^{\prime} x^{\prime} / W_{\mathrm{Q} x}^{d}\right)$. Given a prefix $P_{1} \cdots P_{n}$ of an $L_{2}$-formula, define a sequence of prefixes $\vec{P}_{i}$ recursively as follows. Let $\vec{P}_{0}:=P_{1} \cdots P_{n}$. If $\vec{P}_{i}:=O_{1} \cdots O_{k} \sim O_{k+1} \cdots O_{m}$ and $O_{1} \cdots O_{k}$ is $\sim$-free, define $\vec{P}_{i+1}$ as follows depending on the symbol $O_{k+1}$.

- If $O_{k+1}=\sim$, then $\vec{P}_{i+1}:=O_{1} \cdots O_{k} O_{k+2} \cdots O_{m}$.

- If $O_{k+1}=7$, then $\vec{P}_{i+1}:=O_{1} \cdots O_{k} \rightarrow \sim O_{k+2} \cdots O_{m}$.

- If $O_{k+1}=(\exists x / W)$, then $\vec{P}_{i+1}:=O_{1} \cdots O_{k}(\forall x / W) \sim O_{k+2}^{\star} \cdots O_{m}^{\star}$, where $O_{j}^{\star}=O_{j}$ if $O_{j}$ is one of the two negation symbols, whereas $O_{j}^{\star}=O_{j} \underset{\exists x}{d}$ if $O_{j}$ is a quantifier $(k+2 \leq j \leq m)$. Similarly, if $O_{k+1}=(\forall x / W)$, then $\vec{P}_{i+1}:=O_{1} \cdots O_{k}(\exists x / W) \sim O_{k+2}^{\star} \cdots O_{m}^{\star}$, where $O_{j}^{\star}=O_{j \forall x}^{d}$ if $O_{j}$ if $O_{j}$ is a quantifier, else $O_{j}^{\star}=O_{j}$.

These rules apply until a prefix $O_{1} \cdots O_{r} O_{r+1}$ is reached (in at most $n$ steps) such that $O_{1} \cdots O_{r}$ is $\sim$-free. For example, the rules transform $\sim \forall x(\exists y /\{\forall x\}) R(x, y)$ into $\exists x \sim(\exists y /\{\exists x\}) R(x, y)$, which is transformed into $\exists x(\forall y /\{\exists x\}) \sim R(x, y)$. It is not difficult to verify that $\vec{P}_{i} \chi$ and $\vec{P}_{i+1} \chi$ are strongly equivalent for all quantifierfree formulas $\chi$. 


\section{$7 \quad L_{1}$ and Second-Order Logic}

We prove that our semantics interprets $\rightarrow$ in $L_{1}$ as classical negation, and we establish a strict upper bound to the expressivity of $L_{1}$ as a fragment of SO. In the present section we will restrict attention to $L_{1}$-formulas whose prefix is $\sim$-free; by Fact 6.10 we may do so without loss of generality. We will use known results about the Henkin quantifier logic $L^{*}$ (see Krynicki and Mostowski [19]).

A Henkin quantifier $H \vec{x} \vec{y}$ is an expression of the form

$$
\left(\begin{array}{cccc}
\forall x_{1}^{1} & \ldots & \forall x_{n_{1}}^{1} & \exists y_{1} \\
\vdots & \ddots & \vdots & \vdots \\
\forall x_{1}^{k} & \ldots & \forall x_{n_{k}}^{k} & \exists y_{k}
\end{array}\right),
$$

where $k, n_{i} \geq 1$ for all $i:=1, \ldots, k$ and the $x_{j}^{i}$ and the $y_{l}$ are (object-language) variables. Given a relational vocabulary $\tau$, the syntax of the logic $L^{*}$ is generated by the following grammar:

$$
R\left(x_{1}, \ldots, x_{m}\right)\left|x_{1}=x_{2}\right| \neg \psi|(\psi \vee \psi)|(\psi \wedge \psi)|\forall x \psi| \exists x \psi \mid H \vec{x} \vec{y} \psi,
$$

where $R \in \tau$ is $m$-ary, $x$ and the $x_{i}$ are variables, $\vec{y}$ is a $k$-tuple of variables, and $\vec{x}$ is a $\sum_{i=1}^{k} n_{i}$-tuple of variables with $n_{1}, \ldots, n_{k} \geq 1$. We require that $L^{*}$-formulas be regular: one and the same variable never occurs in nested quantifiers (in particular not in nested Henkin quantifiers). The semantics of $L^{*}$ is obtained by adding to the recursive clauses defining the satisfaction relation of $\mathbf{F O}$ the following clause: $\mathcal{M}, \gamma \models H \vec{x} \vec{y} \psi$ if and only if there are functions $f_{i}: M^{n_{i}} \rightarrow M$ (with $1 \leq i \leq k$ ) such that for all $a_{j}^{i} \in M$ (with $1 \leq i \leq k, 1 \leq j \leq n_{i}$ ) we have $\mathcal{M}, \delta_{\vec{f}, \vec{a}} \models \psi$, where $\delta_{\vec{f}, \vec{a}}$ is the extension of $\gamma$ satisfying $\delta_{\vec{f}, \vec{a}}\left(x_{j}^{i}\right)=a_{j}^{i}$ and $\delta_{\vec{f}, \vec{a}}\left(y_{i}\right)=f_{i}\left(a_{1}^{i}, \ldots, a_{n_{i}}^{i}\right)$. Note that by this semantics, $\mathcal{M}, \gamma \models \neg H \vec{x} \vec{y} \neg \psi$ means that for all functions $f_{i}: M^{n_{i}} \rightarrow M$ (with $1 \leq i \leq k$ ) there are elements $a_{j}^{i} \in M$ (with $1 \leq i \leq k, 1 \leq j \leq n_{i}$ ) such that $\mathcal{M}, \delta_{\vec{f}, \vec{a}} \models \psi$. Write $L_{+}^{*}$ for the fragment of $L^{*}$ consisting of formulas of the form $H \vec{x} \vec{y} \psi$, where $\psi$ is quantifier-free. Write $L_{-}^{*}$ for the fragment of $L^{*}$ consisting of negations of $L_{+}^{*}$-formulas. Enderton [4] and Walkoe [22] showed that $\Sigma_{1}^{1}=L_{+}^{*}$, whence $\Pi_{1}^{1}=L_{-}^{*}$. Enderton [4] proved that $L^{*} \leq \Delta_{2}^{1}$, while M. Mostowski [20] showed that the converse does not hold.

We proceed to prove that $\operatorname{logic} L_{1}$ can be translated into $L^{*}$ : for every $\varphi \in L_{1}$ there is $\psi_{\varphi} \in L^{*}$ such that $\mathcal{M}, \gamma \models_{I I}^{+} \varphi$ if and only if $\mathcal{M}, \gamma \models \psi_{\varphi}$ for all suitable structures $(\mathcal{M}, \gamma)$. Let us first agree on some notation. Suppose that $\vec{P}_{\vec{z}, \vec{v}}$ is a substring of the prefix of an $L_{1}$-formula containing only quantifiers, the existential quantifiers of $\vec{P}_{\vec{z}}, \vec{v}$ being $\left(\exists z_{1} / Z_{1}\right), \ldots,\left(\exists z_{m} / Z_{m}\right)$ and its universal quantifiers $\forall v_{1}, \ldots, \forall v_{n}$, with $\vec{z}=z_{1} \cdots z_{m}$ and $\vec{v}=v_{1} \cdots v_{n}$. For each $1 \leq l \leq m$, let $U_{l}$ be the set of variables $v_{j}$ such that the quantifier $\forall v_{j}$ precedes $\left(\exists z_{l} / Z_{l}\right)$ in the string $\vec{P}_{\vec{z}, \vec{v}}$ but does not belong to the set $Z_{l}$. There may well be variables $v_{j}$ with $v_{j} \in U_{l} \cap U_{l^{\prime}}$ for distinct $l, l^{\prime}$. For all $1 \leq l \leq m$, let $V_{l}:=\left\{v_{j}^{l}: v_{j} \in U_{l}\right\}$. Hence by syntactic criteria the sets of variables $V_{l}$ and $V_{l^{\prime}}$ are disjoint whenever $l, l^{\prime}$ are distinct. For all $1 \leq j \leq n$, let $I_{j}:=\left\{l: v_{j} \in U_{l}\right\}$. Write $\theta\left[\vec{P}_{\vec{z}, \vec{v}}\right]:=\bigwedge_{1 \leq j \leq n, l \in I_{j}} v_{j}=v_{j}^{l}$. Let $H\left[\vec{P}_{\vec{z}, \vec{v}}\right]$ be the Henkin quantifier $H \vec{\alpha} \vec{z}$, where $\vec{\alpha}=\alpha_{1}^{1}, \ldots, \alpha_{n_{m}}^{m}$ is a tuple of metavariables with $\alpha_{l}^{l}, \ldots, \alpha_{n_{l}}^{l}$ standing for the variables in the set $V_{l}$ ordered according to increasing subscripts. If $\chi \in L_{1}$ is 
quantifier-free, let $\chi\urcorner$ be the result of replacing all occurrences of $\sim$ in $\chi$ by $\neg$. With each $L_{1}$-formula $\varphi$, we associate $L^{*}$-formulas $\varphi^{+}$and $\varphi^{-}$to be termed the positive and negative translation of $\varphi$ into $L^{*}$, respectively,

- $\chi^{+}=\chi^{\urcorner}$and $\chi^{-}=\neg \chi^{\urcorner}$if $\chi$ is quantifier-free;

- if $\vec{P}_{\vec{z}, \vec{v}} \vec{O} \chi$ is an $L_{1}$-formula such that $\vec{P}_{\vec{z}, \vec{v}}$ is a string of quantifiers and $\vec{O}$ is either empty or begins with $\rightarrow$, then

$$
\begin{aligned}
& \star\left(\vec{P}_{\vec{z}, \vec{v}} \vec{O} \chi\right)^{+}=H\left[\vec{P}_{\vec{z}, \vec{v}}\right] \forall v_{1} \cdots \forall v_{n}\left(\theta\left[\vec{P}_{\vec{z}, \vec{v}}\right] \supset(\vec{O} \chi)^{+}\right), \\
& \star\left(\vec{P}_{\vec{z}, \vec{v}} \vec{O} \chi\right)^{-}=\neg H\left[\vec{P}_{\vec{z}, \vec{v}}\right] \neg \exists v_{1} \cdots \exists v_{n}\left(\theta\left[\vec{P}_{\vec{z}, \vec{v}}\right] \wedge(\vec{O} \chi)^{-}\right) ; \\
& \bullet(\neg \varphi)^{+}=\varphi^{-} \text {and }(\neg \varphi)^{-}=\varphi^{+} .
\end{aligned}
$$

Lemma 7.1 For all $\varphi \in L_{1}$ and all structures $(\mathcal{M}, \gamma)$, we have $\mathcal{M}, \gamma \models_{I I}^{+} \varphi$ if and only if $\mathcal{M}, \gamma \models \varphi^{+}$and $\mathcal{M}, \gamma \models-{ }_{I I}^{-} \sim \varphi$ if and only if $\mathcal{M}, \gamma \models \varphi^{-}$. In particular, then, $L_{1} \leq L^{*}$.

Proof We prove the claim by induction on the number of $\rightarrow$-signs in the prefix of an $L_{1}$-formula. For the base case of $0 \rightarrow$-signs, suppose that $\chi \in L_{1}$ is quantifierfree. Note that syntactically $\chi \in L_{\mathrm{FO}}$ and $\chi^{\urcorner} \in \mathbf{F O}$ and $\chi^{\urcorner} \in L^{*}$. By the interrelations of the semantics of the logics $L_{1}, L_{\mathrm{FO}}$, FO, and $L^{*}$, we have $\mathcal{M}, \gamma \models_{I I}^{+} \chi$ if and only if $\chi$ is satisfied in $(\mathcal{M}, \gamma)$ according to the semantics of $L_{\mathrm{FO}}$ if and only if $\chi\urcorner$ is satisfied in $(\mathcal{M}, \gamma)$ according to the semantics of FO if and only if $\chi^{+}=\chi^{\urcorner}$ is satisfied in $(\mathcal{M}, \gamma)$ according to the semantics of $L^{*}$. Further, $\mathcal{M}, \gamma \models_{I I}^{-} \sim \chi$ if and only if (Fact 2.1) $\mathcal{M}, \gamma \models F_{I I}^{+} \sim \chi$ if and only if $\sim \chi$ is satisfied in $(\mathcal{M}, \gamma)$ according to the semantics of $L_{\mathrm{FO}}$ if and only if $\left.\chi^{-}=\neg \chi\right\urcorner$ is satisfied in $(\mathcal{M}, \gamma)$ according to the semantics of $L^{*}$. Suppose, then, that the claim holds for formulas with $n \rightarrow$-signs in the prefix. Let $\varphi$ be a formula with $n+1 \rightarrow$-signs. We distinguish two cases.

(1) Suppose $\varphi=\neg \psi$. We have $\mathcal{M}, \gamma \models_{I I}^{+} \rightarrow \psi$ if and only if there is a w.s. for player $I I$ in metagame $\Gamma\left(\psi, \mathcal{M}, \gamma, \rho_{0}^{*},-\right)$ if and only if $\mathcal{M}, \gamma \models_{I I}^{-} \sim \psi$ if and only if (inductive hypothesis) $\mathcal{M}, \gamma \models \psi^{-}$, where $\psi^{-}=(\neg \psi)^{+}$. Further, $\mathcal{M}, \gamma \models_{I I}^{-} \sim \longrightarrow \psi$ if and only if there is a w.s. for player II in metagame $\Gamma\left(\neg \psi, \mathcal{M}, \gamma, \rho_{0}^{*},-\right)$ if and only if $\mathcal{M}, \gamma \models_{I I}^{+} \psi$ if and only if (ind. hyp.) $\mathcal{M}, \gamma \models \psi^{+}$, where $\psi^{+}=(\neg \psi)^{-}$.

(2) Suppose $\varphi=\vec{P}_{\vec{z}, \vec{v}} \vec{O} \chi$, where $\vec{P}_{\vec{z}, \vec{v}}$ is nonempty and $\vec{O}$ is either empty or begins with $\rightarrow$. Consider first the positive translation. Assume $\mathcal{M}, \gamma \models_{I I}^{+} \vec{P}_{\vec{z}, \vec{v}} \vec{O} \chi$. Thus, there is a w.s. for $I I$ in metagame $\Gamma\left(\vec{P}_{\vec{z}, \vec{v}} \vec{O} \chi, \mathcal{M}, \gamma,+\right)$ which yields for every existential quantifier $\left(\exists z_{i} / W_{i}\right)$ in $\vec{P}_{\vec{z}}, \vec{v}$ a local strategy function $f_{i}$ such that against any sequence of moves $\vec{a}$ by $I$ for the universal quantifiers in $\vec{P}_{\vec{z}, \vec{v}}$, we have $\mathcal{M}, \delta \models_{I I}^{+} \quad \vec{O} \chi$, where $\delta$ is the extension of the assignment $\gamma$ determined by $\vec{f}$ and $\vec{a}$. By (1) we have $\mathcal{M}, \delta \models(\vec{O} \chi)^{+}$. Given how the Henkin quantifier $H\left[\vec{P}_{\vec{z}, \vec{v}}\right]$ and the formula $\theta\left[\vec{P}_{\vec{z}, \vec{v}}\right]$ are defined, the local strategy function for $\left(\exists z_{i} / W_{i}\right)$ witnesses the existential quantifier $\exists z_{i}(1 \leq i \leq m)$ in $H\left[\vec{P}_{\vec{z}, \vec{v}}\right]$ so that $\mathcal{M}, \gamma \models H\left[\vec{P}_{\vec{z}, \vec{v}}\right] \forall v_{1} \cdots \forall v_{n}\left(\theta\left[\vec{P}_{\vec{z}, \vec{v}}\right] \supset(\vec{O} \chi)^{+}\right)$. Conversely, if $\mathcal{M}, \gamma \models$ $H\left[\vec{P}_{\vec{z}, \vec{v}}\right] \forall x_{1} \cdots \forall v_{n}\left(\theta\left[\vec{P}_{\vec{z}, \vec{v}}\right] \supset(\vec{O} \chi)^{+}\right)$, let $f_{1}, \ldots, f_{m}$ be the witnesses of $\exists z_{1}, \ldots, \exists z_{m}$ in $H\left[\vec{P}_{\vec{z}, \vec{v}}\right]$ yielding for all values $a_{j} \in M$ of $v_{j}$ with $1 \leq j \leq n$ values of $z_{1}, \ldots, z_{m}$ such that the assignment $\delta_{\vec{f}, \vec{a}}$ extending $\gamma$ hence obtained satisfies $\mathcal{M}, \delta_{\vec{f}, \vec{a}} \models(\vec{O} \chi)^{+}$. By (1) there is a w.s., call it $F_{\vec{f}, \vec{a}}$, for $I I$ in $\Gamma\left(\vec{O} \chi, \mathcal{M}, \delta_{\vec{f}, \vec{a}},+\right)$. 
Define a strategy $F^{\prime}$ in $\Gamma\left(\vec{P}_{\vec{z}, \vec{v}} \vec{O} \chi, \mathcal{M}, \gamma,+\right)$ as follows: choose for each quantifier $\left(\exists z_{i} / W_{i}\right)$ in the string $\vec{P}_{\vec{z}, \vec{v}}$ the function $f_{i}$, and for any tuple of values $\left(a_{1}, \ldots, a_{n}\right)$ chosen for the universal quantifiers $\forall v_{1}, \ldots, \forall v_{n}$ in the string $\vec{P}_{\vec{z}, \vec{v}}$, from the position $\left(\vec{O} \chi, \mathcal{M}, \delta_{\vec{f}, \vec{a}}, \rho_{0},+\right)$ onwards use the strategy $F_{\vec{f}, \vec{a}}$. Clearly $F^{\prime}$ is winning for $I I$.

We must still check the claim concerning the negative translation. Assuming $\mathcal{M}, \gamma \models_{I I}^{-} \sim \vec{P}_{\vec{z}, \vec{v}} \vec{O} \chi$, there is a w.s., call it $F$, for player II in metagame $\Gamma\left(\vec{P}_{\vec{z}, \vec{v}} \vec{O} \chi, \mathcal{M}, \gamma, \rho_{0}^{*},-\right)$. Against any sequence $\vec{f}$ of local strategy functions chosen by player $I$ for the existential quantifiers in $\vec{P}_{\vec{z}, \vec{v}}$, the strategy $F$ determines a value $a_{i}$ for each universal quantifier $\forall v_{i}$ in $\vec{P}_{\vec{z}, \vec{v}}$ such that $\mathcal{M}, \delta \models_{I I}^{-} \sim \vec{O} \chi$, where $\delta$ is the extension of the assignment $\gamma$ determined by $F$ and $\vec{f}$. Thus, by (1) we have $\mathcal{M}, \delta \models(\vec{O} \chi)^{-}$. Given how the Henkin quantifier $H\left[\vec{P}_{\vec{z}, \vec{v}}\right]$ and the formula $\theta\left[\vec{P}_{\vec{z}, \vec{v}}\right]$ are defined, we have $\mathcal{M}, \gamma \models \neg H\left[\vec{P}_{\vec{z}, \vec{v}}\right] \neg \exists v_{1} \cdots \exists v_{n}\left(\theta\left[\vec{P}_{\vec{z}, \vec{v}}\right] \wedge(\vec{O} \chi)^{-}\right)$, since whenever $f_{1}, \ldots, f_{n}$ are functions chosen for the existential quantifiers in $H\left[\vec{P}_{\vec{z}, \vec{v}}\right]$ and $a_{1}, \ldots, a_{n}$ are the values assigned by the strategy $F$ to the universal quantifiers $\forall v_{1}, \ldots, \forall v_{n}$ in $\vec{P}_{\vec{z}, \vec{v}}$, each existential quantifier $\exists v_{i}$ is witnessed by the value $a_{i}$ and each universal quantifier $\forall \alpha_{k}^{r}$ in $H\left[\vec{P}_{\vec{z}}, \vec{v}\right]$ is witnessed by the value $a_{j}$ if $v_{j}^{l}$ is the variable for which the metavariable $\alpha_{k}^{r}$ stands. Conversely, if $\mathcal{M}, \gamma \models \neg H\left[\vec{P}_{\vec{z}, \vec{v}}\right] \neg \exists v_{1} \cdots \exists v_{n}\left(\theta\left[\vec{P}_{\vec{z}, \vec{v}}\right] \wedge(\vec{O} \chi)^{-}\right)$, let $F$ be a map assigning to any tuple $\left(f_{1}, \ldots, f_{m}\right)$ of functions corresponding to $\exists x_{1}, \ldots, \exists x_{m}$ in $H\left[\vec{P}_{\vec{z}, \vec{v}}\right]$ values of the variables $\alpha_{1}^{1}, \ldots, \alpha_{n_{m}}^{m}, v_{1}, \ldots, v_{n}$ so that the resulting assignment $\delta_{F, \vec{f}}$ satisfies $\mathcal{M}, \delta_{F, \vec{f}} \models\left(\theta\left[\vec{P}_{\vec{z}, \vec{v}}\right] \wedge(\vec{O} \chi)^{-}\right)$. In particular, then, by item (1) we have $\mathcal{M}, \delta_{F, \vec{f}} \models_{I I}^{-} \sim \vec{O} \chi$; that is, there is a w.s., call it $F_{\vec{f}}^{\prime}$, for player $I I$ in metagame $\Gamma\left(\vec{O} \chi, \mathcal{M}, \delta_{F, \vec{f}}, \rho_{0}^{*},-\right)$. Clearly the strategy which consists of letting the map $F$ determine the moves for the universal quantifiers in the string $\vec{P}_{\vec{z}, \vec{v}}$ against the adversary's moves $\vec{f}$ for the existential quantifiers therein, and thereafter using the strategy $F_{\vec{f}}^{\prime}$, is a w.s. for player $I I$ in metagame $\Gamma\left(\vec{P}_{\vec{z}, \vec{v}} \vec{O} \chi, \mathcal{M}, \gamma, \rho_{0}^{*},-\right)$. Hence, $\mathcal{M}, \gamma \models-\vec{P}_{\vec{z}, \vec{v}} \vec{O} \chi$.

We are in a position to see that in $L_{1}$ the connective $\rightarrow$ captures classical negation. Further, we see that $L_{1}$ is strictly less expressive than the $\Delta_{2}^{1}$-fragment of SO. Note that the positive translation $\varphi^{+}$of an $L_{1}$-formula $\varphi$ is syntactically in $\Sigma_{k+1}^{1}$ if the number of $\rightarrow$-signs in the prefix of $\varphi$ equals $k$.

Theorem 7.2 Let $\varphi \in L_{1}$.

(a) For all suitable models $\mathcal{M}$ and assignments $\gamma$, we have $\mathcal{M}, \gamma \models_{I I}^{+} \rightarrow \varphi$ if and only if $\mathcal{M}, \gamma \not \forall_{I I}^{+} \varphi$.

(b) $\Sigma_{1}^{1} \cup \Pi_{1}^{1} \leq L_{1}<\Delta_{2}^{1}$.

Proof We begin with item (a). First we prove the following claim: $\varphi^{-} \equiv \neg \varphi^{+}$ for all $\varphi \in L_{1}$. If $\varphi$ is quantifier-free, this is immediate: $\varphi^{-}=\neg \varphi^{\neg}=\neg \varphi^{+}$. Suppose inductively that the claim $\psi^{-} \equiv \neg \psi^{+}$holds for all $L_{1}$-formulas $\psi$ with $n$ $\rightarrow$-signs in the prefix. Let $\varphi$ be a formula with $n+1 \rightarrow$-signs. (1) If $\varphi=\neg \psi$, then $\varphi^{-}=(\neg \psi)^{-}=\psi^{+}$. By the inductive hypothesis, $\psi^{+} \equiv \neg \psi^{-}$. And 
$\neg \psi^{-}=\neg(\neg \psi)^{+}=\neg \varphi^{+}$. (2) If $\varphi=\vec{P}_{\vec{z}, \vec{v}} \vec{O} \chi$, then $\varphi^{-}=\left(\vec{P}_{\vec{z}, \vec{v}} \vec{O} \chi\right)^{-}=$ $\neg H\left[\vec{P}_{\vec{z}, \vec{v}}\right] \neg \exists v_{1} \cdots \exists v_{n}\left(\theta\left[\vec{P}_{\vec{z}, \vec{v}}\right] \wedge(\vec{O} \chi)^{-}\right)$, where $H\left[\vec{P}_{\vec{z}, \vec{v}}\right]$ and $\theta\left[\vec{P}_{\vec{z}, \vec{v}}\right]$ are as defined in connection with the translations $(\cdot)^{+}$and $(\cdot)^{-}$before the statement of Lemma 7.1. The formula $\neg H\left[\vec{P}_{\vec{z}, \vec{v}}\right] \neg \exists v_{1} \cdots \exists v_{n}\left(\theta\left[\vec{P}_{\vec{z}, \vec{v}}\right] \wedge(\vec{O} \chi)^{-}\right)$is, by the inductive hypothesis, equivalent to the formula $\neg H\left[\vec{P}_{\vec{z}, \vec{v}}\right] \neg \exists v_{1} \cdots \exists v_{n}\left(\theta\left[\vec{P}_{\vec{z}, \vec{v}}\right] \wedge \neg(\vec{O} \chi)^{+}\right)$, which again is equivalent to $\neg H\left[\vec{P}_{\vec{z}, \vec{v}}\right] \forall v_{1} \cdots \forall v_{n}\left(\theta\left[\vec{P}_{\vec{z}, \vec{v}}\right] \supset(\vec{O} \chi)^{+}\right)=\neg\left(\vec{P}_{\vec{z}, \vec{v}} \vec{O} \chi\right)^{+}$. Having proven the claim, we have for an arbitrary $L_{1}$-formula $\varphi$ and any structure $(\mathcal{M}, \gamma): \mathcal{M}, \gamma \models_{I I}^{+} \rightarrow \varphi$ if and only if (Lemma 7.1) $\mathcal{M}, \gamma \models(\neg \varphi)^{+}$if and only if $\mathcal{M}, \gamma \models \varphi^{-}$if and only if (above claim) $\mathcal{M}, \gamma \models \neg \varphi^{+}$if and only if $\mathcal{M}, \gamma \forall \varphi^{+}$if and only if (Lemma 7.1) $\mathcal{M}, \gamma \not_{I I}^{+} \varphi$.

Let us, then, move on to item (b). To see that $\Sigma_{1}^{1} \leq L_{1}$, by the result of Enderton and Walkoe it suffices to show that $L_{+}^{*} \leq L_{1}$. Given an $L_{+}^{*}$-formula $\varphi:=H \vec{x} \vec{y} \chi$ with $\vec{x}=x_{1}^{1} \cdots x_{n_{k}}^{k}$ and $\vec{y}=y_{1} \cdots y_{k}$, let $\psi_{\varphi}$ be the $L_{1}$-formula

$$
\forall x_{1}^{1} \cdots \forall x_{n_{k}}^{k}\left(\exists y_{1} / W_{1}\right) \cdots\left(\exists y_{k} / W_{k}\right) \chi,
$$

where $W_{i}=\left\{\forall x_{1}^{1}, \ldots, \forall x_{n_{k}}^{k}\right\} \backslash\left\{\forall x_{1}^{i}, \ldots, \forall x_{n_{i}}^{i}\right\}$ for all $1 \leq i \leq k$. Clearly we have $\mathcal{M}, \gamma \models \varphi$ if and only if $\mathcal{M}, \gamma \models_{I I}^{+} \psi_{\varphi}$ for all suitable structures $(\mathcal{M}, \gamma)$, and we may conclude that $\Sigma_{1}^{1} \leq L_{1}$. It follows that also $\Pi_{1}^{1} \leq L_{1}$. For, suppose that $\zeta$ is a $\Pi_{1}^{1}$-formula. Since $\Sigma_{1}^{1}=L_{+}^{*} \leq L_{1}$, there is a formula $\xi$ of $L_{1}$ such that for all suitable structures $(\mathcal{M}, \gamma)$ we have $\mathcal{M}, \gamma \models \zeta$ if and only if $\mathcal{M}, \gamma \not \forall_{I I}^{+} \xi$ if and only if (by item (a)) $\mathcal{M}, \gamma \models_{I I}^{+} \rightarrow \xi$. Since $\rightarrow \xi \in L_{1}$, we may infer that $\Pi_{1}^{1} \leq L_{1}$. Finally, since $L^{*}<\Delta_{2}^{1}$ by the results of Enderton and M. Mostowski, and $L_{1} \leq L^{*}$ by Lemma 7.1, it follows that $L_{1}<\Delta_{2}^{1}$.

\section{The Place of $L_{2}$ in Type Hierarchy}

8.1 The negation $\rightarrow$ in $L_{2}$ While in $L_{1}$ our semantic framework assigns to $\rightarrow$ the meaning of classical negation, this is not so in the larger context of $L_{2}$.

Theorem 8.1 Let $\varphi \in L_{2}$. If $\mathcal{M}, \gamma \models_{I I}^{+} \neg \varphi$, then $\mathcal{M}, \gamma \not \not_{I I}^{+} \varphi$. However, the converse does not hold in general.

Proof For the positive claim, note that if we had both $\mathcal{M}, \gamma \models_{I I}^{+} \rightarrow \varphi$ and $\mathcal{M}, \gamma \models_{I I}^{+} \varphi$, by Fact 6.5 we would have $\mathcal{M}, \gamma \models_{I I}^{-} \sim \varphi$ and $\mathcal{M}, \gamma \models_{I}^{-} \sim \varphi$, which is impossible: there cannot exist a w.s. for both players in metagame $\Gamma(\sim \varphi, \mathcal{M}, \gamma,-)$. Consider, then, the following $L_{2}$-sentence, to be called $\psi: \exists x \rightarrow(\exists y /\{\exists x\}) x=y$. Let $\mathcal{M}$ be a model of the empty vocabulary with $|M| \geq 2$. If we show that there is no w.s. for $I I$ in either of the games $\Gamma(\psi, \mathcal{M},+)$ or $\Gamma(\sim \psi, \mathcal{M},-)$, the negative claim follows. A strategy for $I I$ in metagame $\Gamma(\psi, \mathcal{M},+)$ is an element $a \in M$ chosen for $\exists x$. If $I$ chooses the same element $a$ for $y$, player $I$ in the role of verifier wins the resulting play. Thus, there is no w.s. for player $I I$ in $\Gamma(\psi, \mathcal{M},+)$. As to metagame $\Gamma(\sim \psi, \mathcal{M},-)$, a strategy for $I I$ in this game is an element $a \in M$ chosen for $\exists y$. Let $b$ be an element of $M$ distinct from $a$. The play in which $I$ in the role of verifier chooses $b$ for $\exists x$ and in which II using her or his strategy picks out $a$ for $\exists y$ terminates, while $I$ has the role of falsifier and is consequently won by $I$. We conclude that there is no w.s. for player $I I$ in $\Gamma(\sim \psi, \mathcal{M},-)$ either.

By Theorem 8.1, we cannot express classical negation of each $L_{2}$-formula $\varphi$ in $L_{2}$ by the formula $\rightarrow \varphi$. This of course does not yet prove that the classical negation 
of $\varphi$ could not have a translation into $L_{2}$; it only shows that the simple syntactic operation of prefixing $\varphi$ by $\rightarrow$ does not provide one. However, below we see that for its expressive power $L_{2}$ equals $\Sigma_{1}^{2}$. Given that $\Sigma_{1}^{2} \neq \Pi_{1}^{2}$, we may then infer that the classical negation of an arbitrary $L_{2}$-formula $\varphi$ is not expressible in $L_{2}$ in the first place.

8.2 Fragment $\Sigma_{1}^{2}$ of third-order logic When a model $\mathcal{M}$ is clear from the context, the elements of the domain $M$ are termed first-order objects. If $n \geq 0$, let $\widetilde{F}_{n}$ be the set of all functions of type $M^{n} \rightarrow M$; its elements are $n$-ary second-order $o b$ jects. Let $\mathcal{F}:=\bigcup_{n<\omega} \mathscr{F}_{n}$. If $n, m, k \geq 0$, let $\mathfrak{F}_{n, m, k}$ be the set of all functions of type $\mathscr{F}^{n} \times M^{m} \rightarrow \mathcal{F}_{k}$; its elements are $(n, m, k)$-ary third-order objects. We write $\mathfrak{F}:=\bigcup_{n, m, k<\omega} \mathfrak{F}_{n, m, k}$. First-order objects are a degenerate case of second-order objects, and second-order objects a degenerate case of third-order objects. In thirdorder logic we have, in addition to individual variables, also $n$-ary second-order and $(n, m, k)$-ary third-order function variables with $n$-ary second-order, respectively, $(n, m, k)$-ary third-order objects as values, for all $n, m, k \geq 0$. Given a set $V$ of first-, second-, and third-order variables, the sets of first- and second-order terms are defined recursively as follows.

- First-order variables are first-order terms, and second-order variables are second-order terms.

- If $\mathrm{s}$ is an $n$-ary second-order term and $t_{1}, \ldots, t_{n}$ are first-order terms, then $\mathrm{s}\left(\mathrm{t}_{1}, \ldots, \mathrm{t}_{n}\right)$ is a first-order term.

- If $\mathrm{F}$ is an $(n, m, k)$-ary third-order variable, $\mathrm{s}_{1}, \ldots, \mathrm{s}_{n}$ are second-order terms, and $t_{1}, \ldots, t_{m}$ are first-order terms, then $F\left(s_{1}, \ldots, s_{n}, t_{1}, \ldots, t_{m}\right)$ is a $k$-ary second-order term.

If $\tau$ is a relational vocabulary, $V_{i}$ is a set of variables of order $i$ (with $i:=1,2,3$ ), and $V=V_{1} \cup V_{2} \cup V_{3}$, then the syntax of third-order logic of vocabulary $\tau$ over $V$, denoted $\mathbf{T O}[\tau, V]$, is given by the following grammar:

$$
\varphi::=\mathbf{a}|\neg \mathbf{a}|(\varphi \wedge \varphi)|(\varphi \vee \varphi)| \forall x \varphi|\exists x \varphi| \forall \mathrm{f} \varphi|\exists \mathfrak{f} \varphi| \forall \mathrm{F} \varphi \mid \exists \mathrm{F} \varphi,
$$

where $\mathbf{a}$ is either a string $R\left(\mathrm{t}_{1}, \ldots, \mathrm{t}_{n}\right)$ for some positive integer $n, n$-ary relation symbol $R \in \tau$, and first-order terms $\mathrm{t}_{1}, \ldots, \mathrm{t}_{n}$ over $V$, or else $\mathbf{a}$ is a string $\mathrm{t}=\mathrm{t}^{\prime}$ for first-order terms $\mathrm{t}, \mathrm{t}^{\prime}$ over $V ; \mathrm{x} \in V_{1} ; \mathrm{f} \in V_{2}$; and $\mathrm{F} \in V_{3}$. If $\xi$ is a variable of order $i$, then $\forall \xi$ and $\exists \xi$ are quantifiers of order $i$.

Given functions $\alpha: V_{1} \rightarrow M$ and $\beta: V_{2} \rightarrow \mathscr{F}$ and $\gamma: V_{3} \rightarrow \mathfrak{F}$ such that $\beta\left(\mathrm{f}_{i}\right) \in \mathscr{F}_{n}$ if $\mathrm{f}_{i}$ is $n$-ary and $\gamma\left(\mathrm{F}_{i}\right) \in \mathfrak{F}_{n, m, k}$ if $\mathrm{F}_{i}$ is $(n, m, k)$-ary, the function $\alpha \cup \beta \cup \gamma$ is an assignment in $\mathcal{M}$. If $\delta$ is an assignment, $\zeta$ is an $\vec{a}$-ary object of order $i$, and $\xi$ is an $\vec{a}$-ary variable of order $i$, we write $\delta(\xi / \zeta)$ for the assignment that agrees with $\delta$ except that $\delta(\xi / \zeta)(\xi)=\zeta$. The definition of the satisfaction relation $\mathcal{M}, \delta \models \varphi$ uses the notion of value $\mathrm{u}^{\delta}$ of a term u under assignment $\delta$, specified for first- and second-order terms as follows:

- $\mathrm{x}^{\delta}=\delta(\mathrm{x})$ and $\mathrm{f}^{\delta}=\delta(\mathrm{f})$,

- $\mathrm{s}\left(\mathrm{t}_{1}, \ldots, \mathrm{t}_{n}\right)^{\delta}=\mathrm{s}^{\delta}\left(\mathrm{t}_{1}^{\delta}, \ldots, \mathrm{t}_{n}^{\delta}\right)$,

- $\mathrm{F}\left(\mathrm{s}_{1}, \ldots, \mathrm{s}_{n}, \mathrm{t}_{1}, \ldots, \mathrm{t}_{m}\right)^{\delta}=\delta(\mathrm{F})\left(\mathrm{s}_{1}^{\delta}, \ldots, \mathrm{s}_{n}^{\delta}, \mathrm{t}_{1}^{\delta}, \ldots, \mathrm{t}_{n}^{\delta}\right)$.

In particular, we define $\mathcal{M}, \delta \models R\left(\mathrm{t}_{1}, \ldots, \mathrm{t}_{n}\right)$ if and only if $\left\langle\mathrm{t}_{1}^{\delta}, \ldots, \mathrm{t}_{n}^{\delta}\right\rangle \in R^{\mathcal{M}}$; $\mathcal{M}, \delta \models \exists f \varphi$ if and only if $\mathcal{M}, \delta(\mathrm{f} / f) \models \varphi$ for some suitable $f \in \mathcal{F}$; and $\mathcal{M}, \delta \models \exists \mathrm{F} \varphi$ if and only if $\mathcal{M}, \delta(\mathrm{F} / F) \models \varphi$ for some suitable $F \in \mathfrak{F}$. 
We define $\Sigma_{1}^{2}[\tau, V]$ as the fragment of $\mathbf{T O}[\tau, V]$ whose formulas are of the form $\exists \mathrm{F}_{1} \cdots \exists \mathrm{F}_{n} \varphi$, where $\varphi$ contains no third-order quantifiers. It is not difficult to see that $\Sigma_{1}^{2}$ is closed under disjunction and conjunction as well as first- and second-order existential and universal quantification. Now, $\Sigma_{1}^{2}$-formulas can be proven to admit the following Skolem form.

Fact 8.2 Every $\Sigma_{1}^{2}$-formula $\varphi$ is equivalent to a formula $\psi_{\varphi}$ of the form $\exists \mathrm{F}_{1} \cdots \exists \mathrm{F}_{n} \forall \mathrm{f}_{1} \cdots \forall \mathrm{f}_{m} \forall \mathrm{x}_{1} \cdots \forall \mathrm{x}_{k} \theta$, where $\theta$ is quantifier-free, and $\varphi$ and $\psi_{\varphi}$ have the same free variables.

A straightforward way of obtaining the Skolem form is as follows. First write the formula in negation normal form. Then eliminate in the resulting formula all occurrences of first- and second-order existential quantifiers, replacing the variables each such quantifier binds by a suitable term $F\left(f_{1}, \ldots, f_{n}, x_{1}, \ldots, x_{m}\right)$; the thirdorder variable $\mathrm{F}$ has the arity $(n, m, k)$, where $k=0$ if the term replaces a first-order variable, while if it replaces a second-order variable, $k$ equals the arity of that variable. Finally, the resulting formula is prefixed by the relevant third-order existential quantifiers. The following specific assumptions can actually be made on formulas in Skolem form.

Fact 8.3 For every $\varphi \in \Sigma_{1}^{2}$ there is a logically equivalent $\psi \in \Sigma_{1}^{2}$ such that the following hold.

(a) For every third-order variable $\mathrm{F}$ in $\psi$, there are pairwise distinct second-order variables $f_{1}, \ldots, f_{m}$ and pairwise distinct first-order variables $x_{1}, \ldots, x_{m}$, $\mathrm{y}_{1}, \ldots, \mathrm{y}_{k}$ such that in all its occurrences, $\mathrm{F}$ appears in the term $\mathrm{F}\left(\mathrm{f}_{1}, \ldots, \mathrm{f}_{m}\right.$, $\left.\mathrm{x}_{1}, \ldots, \mathrm{x}_{n}\right)\left(\mathrm{y}_{1}, \ldots, \mathrm{y}_{k}\right)$ with these same variables in the same order.

(b) For every second-order variable $f$ in $\psi$ which does not appear as an argument of a third-order variable, there are fixed first-order variables $\mathrm{y}_{1}, \ldots, \mathrm{y}_{k}$ such that in all its occurrences, $f$ appears in the term $f\left(y_{1}, \ldots, y_{k}\right)$ with these same variables in the same order.

(c) All third-order variables in $\psi$ are of arity $(n, m, 0)$ for some $n, m \geq 0$.

Proof By Fact 8.2 any $\Sigma_{1}^{2}$-formula may be assumed to be of the form

$$
\exists \mathrm{F}_{1} \cdots \exists \mathrm{F}_{p} \forall \mathrm{f}_{1} \cdots \forall \mathrm{f}_{q} \forall \mathrm{x}_{1} \cdots \forall \mathrm{x}_{r} \theta
$$

with $\theta$ quantifier-free. For (a), we indicate suitable transformation rules which turn formulas in Skolem form into equivalent formulas in Skolem form. We will use $\left(\psi_{1} \rightsquigarrow \psi_{2}\right)$ as an abbreviation of $\left(\operatorname{neg}\left(\psi_{1}\right) \vee \psi_{2}\right)$, where neg $\left(\psi_{1}\right)$ is a negation normal form of $\psi_{1}$; recall that in third-order formulas the negation symbol $\neg$ may only appear in front of atomic formulas.

Claim (a.1) We may assume that third-order variables $\mathrm{F}$ appear only in expressions $\mathrm{F}\left(\mathrm{f}_{1}, \ldots, \mathrm{f}_{m}, \mathrm{x}_{1}, \ldots, \mathrm{x}_{n}\right)\left(\mathrm{x}_{n+1}, \ldots, \mathrm{x}_{n+k}\right)$, where the $\mathrm{f}_{i}$ are second-order and the $\mathrm{x}_{j}$ first-order variables. Namely, we may replace $\forall \overrightarrow{\mathrm{g}} \forall \overrightarrow{\mathrm{y}} \theta\left[\mathrm{F}\left(\mathrm{s}_{1}, \ldots, \mathrm{s}_{m}\right.\right.$, $\left.\left.\mathrm{t}_{1}, \ldots, \mathrm{t}_{n}\right)\left(\mathrm{t}_{n+1}, \ldots, \mathrm{t}_{n+k}\right)\right]$ by the formula

$$
\begin{aligned}
& \forall \overrightarrow{\mathrm{g}} \forall \mathrm{f}_{1} \ldots \forall \mathrm{f}_{m} \forall \overrightarrow{\mathrm{y}} \forall \mathrm{x}_{1} \ldots \forall \mathrm{x}_{n+k} \\
& \quad\left(\chi \rightsquigarrow \theta\left[\mathrm{F}\left(\mathrm{f}_{1}, \ldots, \mathrm{f}_{n}, \mathrm{x}_{1}, \ldots, \mathrm{x}_{k}\right)\left(\mathrm{x}_{n+1}, \ldots, \mathrm{x}_{n+k}\right)\right]\right),
\end{aligned}
$$

where $\chi$ equals $\left(\bigwedge_{1 \leq i \leq m} \forall \vec{z}_{i}\left[\mathrm{f}_{i}\left(\vec{z}_{i}\right)=\mathrm{s}_{i}\left(\vec{z}_{i}\right)\right] \wedge \bigwedge_{1 \leq j \leq n+k} \mathrm{x}_{j}=\mathrm{t}_{j}\right)$. In this formula there are still positive occurrences of first-order universal quantifiers in the 
antecedent of the implication (and therefore negative occurrences in the implication itself). However, a formula of the form $\forall \overrightarrow{\mathrm{h}} \forall \overrightarrow{\mathrm{v}}(\forall \mathrm{x}[\mathrm{f}(\mathrm{x})=\mathrm{s}(\mathrm{x})] \rightsquigarrow \psi)$ is equivalent to the formula $\exists \mathrm{F} \forall \overrightarrow{\mathrm{h}} \forall \overrightarrow{\mathrm{v}} \forall \mathrm{u}(\mathrm{u}=\mathrm{F}(\overrightarrow{\mathrm{h}}, \overrightarrow{\mathrm{v}}) \supset[\mathrm{f}(\mathrm{u}) \neq \mathrm{s}(\mathrm{u}) \vee \psi])$, where $\mathrm{u}$ is a fresh first-order variable. Similar equivalences can be used when the antecedent involves a conjunction of formulas $\forall \vec{x}_{i}\left[f_{i}\left(\vec{x}_{i}\right)=s_{i}\left(\vec{x}_{i}\right)\right]$, the $\vec{x}_{i}$ being tuples of any length.

Claim (a.2) We may assume that if an expression of the form $\mathrm{F}\left(\mathrm{f}_{1}, \ldots, \mathrm{f}_{m}\right.$, $\left.\mathrm{x}_{1}, \ldots, \mathrm{x}_{n}\right)\left(\mathrm{x}_{n+1}, \ldots, \mathrm{x}_{n+k}\right)$ appears in $\theta$, then the $\mathrm{f}_{i}$ are pairwise distinct secondorder variables and the $\mathrm{x}_{j}$ are pairwise distinct first-order variables. As to secondorder variables, if syntactically $\mathrm{f}_{i}=\mathrm{f}_{j}$ with $i<j$, then let $\mathrm{h}$ be a fresh second-order variable of the same arity as $\mathrm{f}_{i}$. We may, first, replace $\forall \vec{g} \forall \vec{y} \theta\left[F\left(f_{1}, \ldots, f_{m}, \overrightarrow{x_{1}}\right)\left(\overrightarrow{x_{2}}\right)\right]$ by the formula

$$
\forall \overrightarrow{\mathrm{g}} \forall \overrightarrow{\mathrm{y}}\left(\forall \vec{z}\left[\mathrm{~h}(\vec{z})=\mathrm{f}_{i}(\vec{z})\right] \rightsquigarrow \theta\left[\mathrm{F}\left(\mathrm{f}_{1}, \ldots, \mathrm{f}_{j-1}, \mathrm{~h}, \mathrm{f}_{j+1}, \ldots, \mathrm{f}_{m}, \overrightarrow{\mathrm{x}_{1}}\right)\left(\overrightarrow{\mathrm{x}_{2}}\right)\right] .\right.
$$

The negative occurrences of the first-order universal quantifier can be eliminated as explained in Claim (a.1). We can get rid of repetitions of first-order variables in the expression $\mathrm{F}\left(\mathrm{f}_{1}, \ldots, \mathrm{f}_{m}, \mathrm{x}_{1}, \ldots, \mathrm{x}_{n}\right)\left(\mathrm{x}_{n+1}, \ldots, \mathrm{x}_{n+k}\right)$ similarly, but without the need to introduce third-order existential quantifiers.

Claim (a.3) We may assume that in any two first-order terms in $\theta$ that contain a given third-order variable $\mathrm{F}$, the same string of second-order variables and the same strings of first-order variables appear. For, if $(\vec{f}, \vec{x}, \vec{y})$ and $\left(\vec{f}^{\prime}, \vec{x}^{\prime}, \vec{y}^{\prime}\right)$ are distinct strings with $\overrightarrow{\mathrm{f}}=\mathrm{f}_{1} \cdots \mathrm{f}_{m}, \overrightarrow{\mathrm{f}}^{\prime}=\mathrm{f}_{1}^{\prime} \cdots \mathrm{f}_{\mathrm{m}}^{\prime}, \overrightarrow{\mathrm{x}}=\mathrm{x}_{1} \cdots \mathrm{x}_{n}, \overrightarrow{\mathrm{x}}^{\prime}=\mathrm{x}_{1}^{\prime} \cdots \mathrm{x}_{\mathrm{n}}^{\prime}, \overrightarrow{\mathrm{y}}=\mathrm{y}_{1} \cdots \mathrm{y}_{k}$, $\overrightarrow{y^{\prime}}=y_{1}^{\prime} \cdots y_{k}^{\prime}$, we may first introduce a new third-order variable $\mathrm{F}^{\prime}$ and replace $\forall \overrightarrow{\mathrm{g}} \forall \overrightarrow{\mathrm{z}} \theta\left[\mathrm{F}(\overrightarrow{\mathrm{f}}, \overrightarrow{\mathrm{x}})(\overrightarrow{\mathrm{y}}), \mathrm{F}\left(\overrightarrow{\mathrm{f}^{\prime}}, \overrightarrow{\mathrm{x}^{\prime}}\right)\left(\overrightarrow{\mathrm{y}^{\prime}}\right)\right]$ by

$$
\exists \mathrm{F}^{\prime} \forall \overrightarrow{\mathrm{g}} \forall \overrightarrow{\mathrm{h}} \forall \overrightarrow{\mathrm{h}^{\prime}} \forall \overrightarrow{\mathrm{z}} \forall \overrightarrow{\mathrm{u}} \forall \overrightarrow{\mathrm{u}^{\prime}} \forall \overrightarrow{\mathrm{v}} \forall \overrightarrow{\vec{v}^{\prime}}\left(\theta\left[\mathrm{F}(\overrightarrow{\mathrm{f}}, \overrightarrow{\mathrm{x}})(\overrightarrow{\mathrm{y}}), \mathrm{F}^{\prime}\left(\overrightarrow{\mathrm{f}}^{\prime}, \overrightarrow{\mathrm{x}^{\prime}}\right)\left(\overrightarrow{\mathrm{y}^{\prime}}\right)\right] \wedge \chi\right),
$$

where $\chi$ equals $\left[\left(\bigwedge_{1 \leq i \leq m} \forall \vec{w}_{i}\left[\mathrm{~h}_{i}\left(\vec{w}_{i}\right)=\mathrm{h}_{\mathrm{i}}^{\prime}\left(\vec{w}_{i}\right)\right] \wedge \bigwedge_{1 \leq i \leq n} \mathrm{u}_{i}=\mathrm{u}_{\mathrm{i}}^{\prime} \wedge \bigwedge_{1 \leq i \leq k} \mathrm{v}_{i}=\right.\right.$ $\left.\left.v_{i}^{\prime}\right) \rightsquigarrow F(\vec{h}, \vec{u})(\vec{v})=F^{\prime}\left(\vec{h}^{\prime}, \vec{u}^{\prime}\right)\left(\overrightarrow{v^{\prime}}\right)\right]$ and the arity of $h_{i}$ equals the arity of $f_{i}$, the arity of $\mathrm{h}_{\mathrm{i}}^{\prime}$ equals the arity of $\mathrm{f}_{\mathrm{i}}^{\prime}$, and the variables $\mathrm{h}_{\mathrm{i}}, \mathrm{h}_{\mathrm{i}}^{\prime}, \mathrm{u}_{\mathrm{j}}, \mathrm{u}_{\mathrm{j}}^{\prime}, \mathrm{v}_{\mathrm{l}}, \mathrm{v}_{\mathrm{l}}^{\prime}$ are fresh. Then we may again get rid of negative occurrences of first-order universal quantifiers in the implication as in connection with Claim (a.1).

We have just proven item (a). Using similar equivalences we can prove item (b). For item (c), if $\mathrm{F}^{\prime}$ is an $(m, n+k, 0)$-ary variable, formulas $\exists \mathrm{F} \forall \overrightarrow{\mathrm{f}} \overrightarrow{\mathrm{x}} \overrightarrow{\mathrm{y}} \theta[\mathrm{F}(\overrightarrow{\mathrm{f}}, \overrightarrow{\mathrm{x}})(\overrightarrow{\mathrm{y}})]$ and $\exists \mathrm{F}^{\prime} \forall \overrightarrow{\mathrm{f}} \overrightarrow{\mathrm{x}} \overrightarrow{\mathrm{y}} \theta\left[\mathrm{F}^{\prime}(\overrightarrow{\mathrm{f}}, \overrightarrow{\mathrm{x}}, \overrightarrow{\mathrm{y}})\right]$ are equivalent. Namely, if $F$ is a witness of $\mathrm{F}$, then $F^{\prime}$ defined as follows is a witness of $\mathrm{F}^{\prime}: F^{\prime}(\vec{f}, \vec{a}, \vec{b})=F(\vec{f}, \vec{a})(\vec{b})$ for all suitable tuples $\vec{f}, \vec{a}$, and $\vec{b}$. Conversely, if $F^{\prime}$ is a witness of $F^{\prime}$, for fixed $\vec{h}, \vec{c}$ define first $g_{\vec{h}, \vec{c}}$ as the $k$-ary second-order object satisfying $g_{\vec{h}, \vec{c}}(\vec{b})=F^{\prime}(\vec{h}, \vec{c}, \vec{b})$ for all suitable $\vec{b}$; then define $F$ as follows: $F(\vec{f}, \vec{a})=g_{\vec{f}, \vec{a}}$ for all suitable $\vec{f}, \vec{a}$.

8.3 From $L_{2}$ to $\Sigma_{1}^{2}$ We prove that logic $L_{2}$ can be translated into $\Sigma_{1}^{2}$.

Fact 8.4 There is a translation of $L_{2}$ into $\Sigma_{1}^{2}$.

Proof Let $\psi:=P_{1} \cdots P_{n} \chi$ be an $L_{2}$-formula. By Fact 6.10 we may suppose without loss of generality that the prefix contains no $\sim$-sign. Further, since we are interested in the satisfaction conditions of $\psi$, by Fact 6.7 we may assume that 
$P_{1} \cdots P_{n}=P_{1}^{+} \cdots P_{n}^{+}$. Write $k$ for the number of $\rightarrow$-signs in the prefix. If $k=0, \psi$ is a formula of $L_{\mathrm{IF}}$ and therefore trivially equivalent to a $\Sigma_{1}^{2}$-formula. If $k>0$, there are strings $\vec{O}_{i}$ of quantifiers (with $0 \leq i \leq k+1$ ) such that $P_{1} \cdots P_{n}=\vec{O}_{1} \rightarrow \vec{O}_{2} \cdots \rightarrow \vec{O}_{k} \rightarrow \vec{O}_{k+1}$. Since any $L_{2}$-formula $\rightarrow \rightarrow \psi$ is strongly equivalent to the formula $\psi$, we may assume that the strings $\vec{O}_{2}, \ldots, \vec{O}_{k}$ are nonempty. By contrast, the strings $\vec{O}_{1}$ and $\vec{O}_{k+1}$ may be empty.

- If $i$ is odd, write $\mathbf{A}_{i}$ for the set of universal quantifiers of the string $\vec{O}_{i}$. Let $\forall x_{s_{1}}, \ldots, \forall x_{s_{K}}$ be the list of all elements of $\bigcup_{i} \mathbf{A}_{i}$. In the relevant game, player $I$ chooses an element of the domain for each $\forall x_{s_{j}}$.

- If $i$ is even, write $\mathbf{B}_{i}$ for the set of existential quantifiers of the string $\vec{O}_{i}$. Player $I$ chooses a local strategy function for each of these quantifiers. For every $\left(\exists x_{j} / W_{j}\right) \in \mathbf{B}_{i}$, introduce a term $f_{j}\left(x_{i_{1}}, \ldots, x_{i_{a_{j}}}\right)$, where $\mathrm{f}_{j}$ is an $a_{j}$-ary second-order variable and $x_{i_{1}}, \ldots, x_{i_{a_{j}}}$ are those first-order variables that are bound by a universal quantifier preceding the quantifier $\left(\exists x_{j} / W_{j}\right)$ in the string $\vec{O}_{i}$ but not belonging to the set $W_{j}$. Let $f_{j_{1}}, \ldots, f_{j_{N}}$ be the list of all second-order function variables hence introduced by quantifiers $\left(\exists x_{j} / W_{j}\right) \in \bigcup_{i} \mathbf{B}_{i}$.

- If $i$ is even, write $\mathbf{C}_{i}$ for the set of universal quantifiers of the string $\vec{O}_{i}$. Player II chooses an element of the domain for each of these quantifiers. For every $\left(\forall x_{j} / W_{j}\right) \in \mathbf{C}_{i}$, introduce a term $\mathrm{F}_{j}\left(\mathrm{f}_{i_{1}}, \ldots, \mathrm{f}_{i_{n_{j}}}, x_{r_{1}}, \ldots, x_{r_{m_{j}}}\right)$ satisfying the following conditions. First, $\mathrm{f}_{i_{1}}, \ldots, \mathrm{f}_{i_{n_{j}}}$ are the second-order function variables introduced for those existential quantifiers that (a) appear in a string $\vec{O}_{k}$ with $k \leq i$ for an even number $k$, and (b) do not belong to the set $W_{j}$. Note that among them there are automatically all existential quantifiers of the string $\vec{O}_{i}$, also those coming syntactically after $\left(\forall x_{j} / W_{j}\right)$. Second, $x_{r_{1}}, \ldots, x_{r_{m_{j}}}$ are the first-order variables bound by a universal quantifier which (a) appears in a string $\vec{O}_{k}$ with $k<i$ for an odd number $k$, but (b) does not belong to $W_{j}$. Let $\mathrm{F}_{t_{1}}, \ldots, \mathrm{F}_{t_{H}}$ be the list of all third-order function variables thus introduced by quantifiers in $\bigcup_{i} \mathbf{C}_{i}$.

- If $i$ is odd, write $\mathbf{D}_{i}$ for the set of existential quantifiers of the string $\vec{O}_{i}$. Player II chooses a local strategy function for each of these quantifiers. For every $\left(\exists x_{j} / W_{j}\right) \in \mathbf{D}_{i}$, introduce a term $\mathrm{F}_{j}\left(\mathrm{f}_{i_{1}}, \ldots, \mathrm{f}_{i_{n_{j}}}, x_{r_{1}}, \ldots, x_{r_{m_{j}}}\right)$ satisfying the following. First, $\mathrm{f}_{i_{1}}, \ldots, \mathrm{f}_{i_{n_{j}}}$ are the second-order function variables introduced for those existential quantifiers that (a) appear in a string $\vec{O}_{k}$ with $k<i$ for an even number $k$, and (b) do not belong to the set $W_{i}$. Second, $x_{r_{1}}, \ldots, x_{r_{m_{j}}}$ are the first-order variables that are bound by a universal quantifier which (a) appears in a string $\vec{O}_{k}$ with $k<i$ for an odd number $k$, but (b) does not belong to the set $W_{j}$. Let $\mathrm{F}_{k_{1}}, \ldots, \mathrm{F}_{k_{M}}$ be the list of all third-order function variables thus introduced by quantifiers in $\bigcup_{i} \mathbf{D}_{i}$.

Let $\chi^{*}$ be the result of replacing in $\chi$ every variable $x_{j}$ such that either $\left(\forall x_{j} / W_{j}\right) \in$ $\bigcup_{i} \mathbf{C}_{i}$ or $\left(\exists x_{j} / W_{j}\right) \in \bigcup_{i} \mathbf{D}_{i}$ by the corresponding term $\mathrm{F}_{j}\left(\mathrm{f}_{i_{1}}, \ldots, \mathrm{f}_{i_{n_{j}}}\right.$, $\left.\mathrm{x}_{r_{1}}, \ldots, \mathrm{x}_{r_{m_{j}}}\right)$. Let $\chi^{+}$be the result of first replacing in $\chi^{*}$ every variable $x_{j}$ such that $\left(\exists x_{j} / W_{j}\right) \in \bigcup_{i} \mathbf{B}_{i}$ by the term $\mathrm{f}_{j}\left(\mathrm{x}_{i_{1}}, \ldots, \mathrm{x}_{i_{a_{j}}}\right)$ and then replacing in the resulting string all occurrences of $\sim$ by $\neg$. Let $\varphi_{\psi}$ be the $\Sigma_{1}^{2}$-formula 
$\exists \mathrm{F}_{t_{1}} \cdots \exists \mathrm{F}_{t_{H}} \exists \mathrm{F}_{k_{1}} \cdots \exists \mathrm{F}_{k_{M}} \forall \mathrm{f}_{j_{1}} \cdots \forall \mathrm{f}_{j_{N}} \forall \mathrm{x}_{s_{1}} \cdots \forall \mathrm{x}_{s_{K}} \pm \chi^{*}$, where $\pm \chi^{*}=\chi^{*}$ if the number $k$ is even, and $\pm \chi^{*}=\neg \chi^{*}$ if $k$ is odd. Now, given a model $\mathcal{M}$ and an assignment $\gamma$ over the free variables of $\psi$, we clearly have that $\left(F_{t_{1}}, \ldots, F_{t_{H}}, F_{k_{1}}, \ldots, F_{k_{M}}\right)$ is a w.s. for $I I$ in metagame $\Gamma(\mathcal{M}, \psi, \gamma,+)$ if and only if $\mathcal{M}, \delta_{\gamma} \models \forall \mathrm{f}_{j_{1}} \cdots \forall \mathrm{f}_{j_{N}} \forall \mathrm{x}_{s_{1}} \cdots, \forall \mathrm{x}_{s_{K}} \pm \chi^{*}$, where $\delta_{\gamma}$ is an extension of $\gamma$ satisfying $\delta_{\gamma}\left(\mathrm{F}_{i}\right)=F_{i}$ for all $i \in\left\{t_{1}, \ldots, t_{H}, k_{1}, \ldots, k_{M}\right\}$.

8.4 From $\Sigma_{1}^{2}$ to $L_{2}$ We prove that conversely, $\Sigma_{1}^{2}$ is translatable into $L_{2}$.

Theorem 8.5 There is a translation of $\Sigma_{1}^{2}$ into $L_{2}$.

Proof Let $\psi \in \Sigma_{1}^{2}$ be arbitrary. By Facts 8.2 and 8.3, $\psi$ may be assumed to be of the form $\exists \mathrm{F}_{1} \cdots \exists \mathrm{F}_{n} \forall \mathrm{f}_{1} \cdots \forall \mathrm{f}_{m} \forall \mathrm{x}_{1} \cdots \forall \mathrm{x}_{k} \theta$, where the first-order terms satisfy the conditions (a), (b), and (c) laid down in Fact 8.3, and $\theta$ is quantifier-free. Let $\varphi_{\psi}$ be the $L_{2}$-formula

$$
\begin{aligned}
& \forall x_{1} \cdots \forall x_{k} \rightarrow \forall y_{1} \cdots \forall y_{k}\left(\exists z_{1} / W_{1}\right) \cdots\left(\exists z_{m} / W_{m}\right) \\
& \rightarrow\left(\exists t_{1} / V_{1}\right) \cdots\left(\exists t_{n} / V_{n}\right)\left(\chi \wedge \theta^{*}\right),
\end{aligned}
$$

where the sets $W_{i}$ and $V_{l}$ and the formulas $\chi$ and $\theta^{*}$ are as follows. First, for every second-order function variable $f_{i}$ in $\theta$, if $x_{j_{1}}, \ldots, x_{j_{i}}$ are the pairwise distinct first-order variables such that all those occurrences of $f_{i}$ in $\theta$ that do not occur as arguments of a third-order variable appear in the expression $\mathrm{f}_{i}\left(\mathrm{x}_{j_{1}}, \ldots, \mathrm{x}_{\mathrm{r}_{i}}\right)$, then let $\mathbf{X}_{i}:=\left\{\mathrm{x}_{j_{1}}, \ldots, \mathrm{x}_{j_{r_{i}}}\right\}$. For every third-order function variable $\mathrm{F}_{i}$ in $\theta$, if $\mathrm{f}_{j_{1}}, \ldots, \mathrm{f}_{j_{s_{i}}}, \mathrm{x}_{j_{1}^{\prime}}, \ldots, \mathrm{x}_{j_{s_{i}^{\prime}}}$ are the pairwise distinct variables such that all occurrences of $F_{i}$ in $\theta$ appear in the expression $F_{i}\left(f_{j_{1}}, \ldots, f_{j_{s_{i}}}, x_{j_{1}^{\prime}}, \ldots, x_{j_{s_{i}^{\prime}}^{\prime}}\right)$, then let $\mathbf{Y}_{i}:=\left\{\mathrm{f}_{j_{1}}, \ldots, \mathrm{f}_{j_{i}}\right\}$ and $\mathbf{Z}_{i}:=\left\{\mathrm{x}_{j_{1}^{\prime}}, \ldots, \mathrm{x}_{j_{s_{i}^{\prime}}}\right\}$. For all $1 \leq i \leq m$ and $1 \leq l \leq n$, we set

- $W_{i}:=\left\{\forall y_{j}: 1 \leq j \leq k\right.$ and $\left.x_{j} \notin \mathbf{X}_{i}\right\}$

- $V_{l}:=\left\{\exists z_{j}: 1 \leq j \leq m\right.$ and $\left.\mathrm{f}_{j} \notin \mathbf{Y}_{l}\right\} \cup\left\{\forall x_{j}: 1 \leq j \leq k\right.$ and $\left.x_{j} \notin \mathbf{Z}_{l}\right\}$;

- $\chi:=\bigwedge_{1 \leq i \leq k} x_{i}=y_{i}$;

- $\theta^{*}$ is the result of first replacing in $\theta$, for all $1 \leq i \leq m$ and $1 \leq l \leq n$, every occurrence of the term $\mathrm{f}_{i}\left(\mathrm{x}_{j_{1}}, \ldots, \mathrm{x}_{j_{r_{i}}}\right)$ by the variable $z_{i}$ and every occurrence of the term $\mathrm{F}_{l}\left(\mathrm{f}_{j_{1}}, \ldots, \mathrm{f}_{j_{s_{l}}}, \mathrm{x}_{j_{1}^{\prime}}, \ldots, \mathrm{x}_{j_{s_{l}^{\prime}}}\right)$ by the variable $t_{l}$, and then replacing in the resulting string all occurrences of $\neg$ by $\sim$.

Note that $\left(\chi \wedge \theta^{*}\right)$ is a quantifier-free formula which does not contain second- or third-order variables. Indeed, it is a quantifier-free $L_{\mathrm{FO}}$-formula. Consequently $\varphi_{\psi}$ is an $L_{2}$-formula. Let, then, $(\mathcal{M}, \gamma)$ be any suitable structure. If $\delta_{\gamma}$ is an extension of $\gamma$ providing witnesses for the existential quantifiers $\exists \mathrm{F}_{1}, \ldots, \exists \mathrm{F}_{n}$ so that $\mathcal{M}, \delta_{\gamma} \models \forall \mathrm{f}_{1} \ldots \forall \mathrm{f}_{m} \forall \mathrm{x}_{1} \cdots \forall \mathrm{x}_{k} \theta$, then clearly the sequence $\left(F_{\forall y_{1}}, \ldots, F_{\forall y_{k}}\right.$, $\left.F_{\exists t_{1}}, \ldots, F_{\exists t_{n}}\right)$ defined as follows is a w.s. for $I I$ in metagame $\Gamma\left(\mathcal{M}, \varphi_{\psi}, \gamma,+\right)$ : $F_{\forall y_{j}}$ assigns to $y_{j}$ the same value that player $I$ has earlier assigned to $x_{j}$, and $F_{\exists t_{i}}=\delta_{\gamma}\left(\mathrm{F}_{i}\right)$. Conversely, if $F_{\exists t_{1}}, \ldots, F_{\exists t_{n}}$ are the strategy functions for the quantifiers $\left(\exists t_{1} / V_{1}\right), \ldots,\left(\exists t_{n} / V_{n}\right)$ belonging to a w.s. for $I I$ in $\Gamma\left(\mathcal{M}, \varphi_{\psi}, \gamma,+\right)$, then clearly $\mathcal{M}, \delta_{\gamma} \models \forall f_{1} \ldots \forall f_{m} \forall x_{1} \ldots \forall x_{k} \theta$, given that $\delta_{\gamma}$ is the extension of $\gamma$ satisfying $\delta_{\gamma}\left(\mathrm{F}_{\mathrm{i}}\right)=F_{\exists t_{i}}$. 


\section{Corollary 8.6}

(a) $L_{2}=\Sigma_{1}^{2}$;

(b) classical negation is not expressible in $L_{2}$.

Proof Item (a) is immediate from Fact 8.4 and Theorem 8.5. Item (b) follows by the fact that $\Sigma_{1}^{2}$ is not closed under complementation.

Extending $L_{\mathrm{FO}}$ by allowing independence indications as in $L_{\mathrm{IF}}$ yields a logic with the expressive power of $\Sigma_{1}^{1}$. Observe that by Corollary 8.6, liberalizing the slashing conventions of $L_{1}$ so as to obtain $L_{2}$ yields an even more dramatic increase in expressive power: from a logic less expressive than $\Delta_{2}^{1}$, a logic is obtained that is more expressive than the full second-order logic.

\section{Conclusion}

Let us say that a semantic game is of order 1, if all model-related entities with which the players operate are individuals. Given a fragment $X$ of higher-order logic, we can ask whether a logic $L_{X}$ can be found whose semantic games are of order 1 and which has the same expressive power as $X$. It is not entirely trivial to find out whether for a given $X$ such a logic $L_{X}$ exists. It took for instance some effort to find out that the fragment $\Sigma_{1}^{2}$ of $\mathbf{T O}$ is of this kind.

We provided a game-theoretical interpretation to the connective $\rightarrow$, which yielded to $\rightarrow$ the meaning of classical negation relative to logic $L_{1}$. This was achieved by enriching positions of semantic games by an additional component-a mode. The import of $\rightarrow$ on the play level is actually the same as that of $\sim$. However, through effecting a mode change, it has important repercussions at the strategy level. As mentioned in Section 1, Hintikka has repeatedly claimed that classical negation does not admit a game-theoretic interpretation. Our semantic games show that this claim does not categorically hold if relatively small modifications in game rules are allowed. Earlier a way of capturing classical negation using 3-player strategic games was found by Figueira, Gorín, and Grimson [6] (see Section 9.3 below). Hintikka could not object to our formulation of semantic games on the basis that we interpret $\rightarrow$ as acting on the strategy level. For, this is precisely how Hintikka himself has interpreted the independence indications in $L_{\mathrm{IF}}$ : they regulate the players' strategies but have no bearing at the play level. By contrast, the games used by Figueira, Gorín and Grimson differ from Hintikka's semantic games in several respects.

9.1 Hintikka's subgame semantics The only way in which Hintikka has considered it possible to deal with classical negation is via the following strategy-level rule with a limited range of application: there is a w.s. for $I I$ in game $G(\neg \varphi, \mathcal{M}, \gamma)$ if and only if there is no w.s. for $I I$ in $G(\varphi, \mathcal{M}, \gamma)$. He has usually limited attention to the case where $\varphi$ is a sentence, but in $L_{\mathrm{FeIF}}$ (see [10]) he allows any formulas subject to the condition that if $(\mathrm{Q} x / W)$ is a quantifier in the syntactic scope of an occurrence of $\neg$, then all quantifiers referred to via the set $W$ are likewise in the syntactic scope of this occurrence of $\neg$. Thus, formulas like $\forall x \neg(\exists y /\{\forall x\}) R(x, y)$ are excluded. Apart from denoting the classical negation by $\neg$ rather than by $\neg$, the $L_{\mathrm{FeIF}}$-formulas in prenex form are exactly the formulas of $L_{1}$. Hintikka rules out the possibility of finding an interpretation to formulas not complying with the mentioned syntactic constraint-such as those in $L_{2} \backslash L_{1}$. In order to conceptualize the use of the strategy-level rule for $\neg$ in $L_{\mathrm{FeIF}}$, Hintikka [9], [10] resorts to the idea of 
semantic games with subgames (see Carlson and Hintikka [3], Hintikka and Kulas [11]). Hintikka or his associates have not developed this notion in full formal detail, but the basic idea is clear. In such games, the continuation of a given play may require having played an entire smaller "game" (a subgame), meaning that the players have selected corresponding strategy functions. Hence there is, for example, a w.s. for $I I$ in game $G(\neg \forall x \exists y \neg \exists z R(x, y, z), \mathcal{M})$ if and only if $I I$ lacks a w.s. in subgame $G(\forall x \exists y \neg \exists z R(x, y, z), \mathcal{M})$. There would be a w.s. for $I I$ in the subgame if and only if there was a map $f: M \rightarrow M$ such that for all $a \in M$, player $I I$ lacks a w.s. in subgame $G(\exists z R(x, y, z), \mathcal{M}, x \mapsto a, y \mapsto f(a))$, that is, $\langle a, f(a), b\rangle \notin R^{\mathcal{M}}$ for all $b \in M$. It is a part of the subgame semantics idea that the moves of player $j$ in later subgames are allowed to depend on the strategy functions chosen by $j$ 's adversary in earlier subgames. We see that a w.s. for $I I$ in the sample game would be a pair of functionals $\left(F_{x}, F_{z}\right)$ such that if $f: M \rightarrow M$ is a function determining a value of $y$ depending on a value of $x$, then $F_{x}(f)$ is a value of $x$, and $F_{z}(f)$ is a value of $z$, and $\left\langle F_{x}(f), f\left(F_{x}(f)\right), F_{z}(f)\right\rangle \in R^{\mathcal{M}}$. Using the notion of mode, we were able to formulate semantic games for $L_{2}$ which on the play level involve sequences of moves of the same kind as in plain first-order semantic games. What Hintikka achieves with subgames, we achieve with metagames, used for singling out the sorts of strategy-level attributes that we are interested in. Hintikka's subgame semantics for $L_{1}$ agrees with the semantics we have assigned to this logic. Incidentally, we saw in Theorem 7.2 that $L_{1}<\Delta_{2}^{1}$. This contradicts Hintikka's argument in [10] to the effect that $L_{\text {FeIF }}$ has the expressive power of full second-order logic. This may be contrasted with the case of team logic (see Väänänen [21]), the result of extending dependence logic with classical negation, which in fact has the expressive power of SO (see Kontinen and Nurmi [17]).

9.2 Flattening and Hodges's compositional semantics Hodges [12], [13] formulated a compositional semantics to $L_{\mathrm{IF}}$ by defining the relations $\mathcal{M} \models_{X}^{t} \varphi$ ( $X$ is a trump for $\varphi$ in $\mathcal{M})$ and $\mathcal{M} \models_{X}^{\cot } \varphi(X$ is a cotrump for $\varphi$ in $\mathcal{M})$ recursively on the structure of $L_{\mathrm{IF}}$-subformulas $\varphi .^{3}$ Here $X$ is a set of assignments, and $\varphi$ may have arbitrary free atomic or independence variables. Dual negation is interpreted via the clause $\mathcal{M} \models_{X}^{t} \sim \varphi$ if and only if $\mathcal{M} \models_{X}^{\text {cot }} \varphi$. Hodges's semantics captures the game-theoretic semantics for $L_{\mathrm{IF}}$ : for every formula $\varphi \in L_{\mathrm{IF}}$ (by definition without free independence variables), there is a w.s. for player $I I$ in $G(\varphi, \mathcal{M}, \gamma)$ if and only if $\mathcal{M}_{\{\gamma\}}^{t} \varphi$. Hodges considered also an extended language having available the flattening operator $\downarrow$ with the following semantics:

- $\mathcal{M} \models_{X}^{t} \downarrow \varphi$ if and only if $X$ is nonempty and for every $\gamma \in X$ we have $\mathcal{M} \models_{\{\gamma\}}^{t} \varphi$

- $\mathcal{M} \models_{X}^{\text {cot }} \downarrow \varphi$ if and only if $X$ is nonempty and for every $\gamma \in X$ we have $\mathcal{M} \forall_{\{\gamma\}}^{t} \varphi$.

The idea behind the semantics of $\downarrow$ is this. Independence indications impose uniform choices to be made relative to a multitude of possible variable assignments. Thus, the semantic effect of independence indications is void when the evaluation is relative to a single assignment. If $\psi$ is a subformula with free independence variables, let the "flattening of $\psi$ " be the formula $\psi^{f}$ without free independence variables, obtained from $\psi$ by removing from its independence indications all quantifiers $Q x$ with $x$ free. Whenever $X_{0}$ is a singleton, we have $\mathcal{M} \models_{X_{0}}^{t} \varphi$ if and only if $\mathcal{M} \models_{X_{0}}^{t} \varphi^{f}$, 
and $\mathcal{M} \models \models_{X_{0}}^{\cot } \varphi$ if and only if $\mathcal{M} \models_{X_{0}}^{\cot } \varphi^{f}$. Therefore the formulas $\downarrow \varphi$ and $\downarrow \varphi^{f}$ have the same trumps and the same cotrumps, and the semantic effect of applying the flattening operator $\downarrow$ is that the formula to which it is applied will be treated as if it had no free independence variables. Hodges proposed to define $\neg$ by stipulating that $\neg \varphi$ means $\sim \downarrow \varphi$.

Regarding "intuitions" on classical negation in the IF-logical setting, Hodges actually agrees with Hintikka, who blocks at the outset syntactic contexts like $\forall x \neg(\exists y / W)$ with $\forall x \in W$. Hodges allows such contexts, but his semantics takes care that independence indications crossing a classical negation sign are vacuous. For example, $\mathcal{M} \models^{t} \forall x \neg(\forall y /\{\forall x\}) R(x, y)$ if and only if for all $a \in M$ we have $\mathcal{M} \models_{\{x \mapsto a\}}^{t} \sim \downarrow(\forall y /\{\forall x\}) R(x, y)$, that is, $\mathcal{M} \models_{\{x \mapsto a\}}^{\cot } \downarrow(\forall y /\{\forall x\}) R(x, y)$, that is, $\mathcal{M} \forall_{\{x \mapsto a\}}^{t}(\forall y /\{\forall x\}) R(x, y)$, that is, $\mathcal{M} \forall_{\{x \mapsto a\}}^{t} \forall y R(x, y)$, that is, $\mathcal{M} \models_{\{x \mapsto a\}}^{t} \neg \forall y R(x, y)$. Thus, $\forall x \neg(\forall y /\{\forall x\}) R(x, y)$ and $\forall x \neg \forall y R(x, y)$ are truth-equivalent on Hodges's semantics. This is to be contrasted with our treatment of $L_{2}$ in which the independence indications crossing a $\rightarrow$-sign have a perfectly nonvacuous interpretation in the metagame setting. On our semantics $\forall x \rightarrow(\forall y /\{\forall x\}) R(x, y)$ is actually truth-equivalent to $\exists y \forall x \rightarrow R(x, y)$.

9.3 Capturing classical negation game-theoretically Hodges [12] did not attempt to phrase the semantics of $\downarrow$ game-theoretically, and neither did he comment on the expressive power of the language obtained by having $\downarrow$ available in the IF-like logic he formulated. Both of these endeavors are undertaken by Figueira, Gorín, and Grimson [5], [6]. Write $L(\downarrow)$ for the set of formulas obtained from $L_{\mathrm{IF}}$-formulas by allowing arbitrary occurrences of $\downarrow$ in the prefix. Observe that consequently formulas of $L(\downarrow)$ are in prenex form, they are regular, and they contain no free independence variables. It follows from [6, Theorem 2] that $L(\downarrow)$ has the same expressive power as the logic $\operatorname{SL}(\downarrow)$ discussed by the mentioned authors, if we restrict attention to $\operatorname{SL}(\downarrow)$-formulas whose all free variables appear in atomic subformulas. In [5] Figueira, Gorín, and Grimson associate with all $L(\downarrow)$-formulas and all models a 3-player game in strategic form; their original motivation for defining such games was to find a semantic analysis of IF-logical formulas avoiding certain problems related to renaming of variables (see Janssen [16]). If the players are $I, I I$, and $I I I$, then in each turn both players $I$ and II select functions reminiscent of local strategies in our metagames, whereafter player III carries out a certain sort of evaluation. With $k$ nested $\downarrow$-signs, there are $k$ turns in the correlated game. The authors show that for $L(\downarrow)$-formulas, Hodges's compositional semantics and their game semantics coincide. In [6], the authors prove, utilizing their game-theoretically formulated semantics, that $L(\downarrow) \leq \Delta_{2}^{1}$. We may note that actually $L_{1}=L(\downarrow)$ : for every $\varphi \in L_{1}$ there is $\psi_{\varphi} \in L(\downarrow)$ such that $\mathcal{M}, \gamma \models_{I I}^{+} \varphi$ if and only if $\mathcal{M} \models_{\{\gamma\}}^{t} \psi_{\varphi}$; and for every $\psi \in L(\downarrow)$ there is $\varphi_{\psi} \in L_{1}$ such that $\mathcal{M} \models_{\{\gamma\}}^{t} \psi$ if and only if $\mathcal{M}, \gamma \models_{I I}^{+} \varphi_{\psi}$. That $L_{1} \leq L(\downarrow)$ follows because in $L_{1}$ the connective $\rightarrow$ has the force of classical negation, and when applied to a formula without free independence variables, the combination $\sim \downarrow$ likewise has the force of classical negation. By the semantics of $\sim$ and by what is observed above about flattening, any $L(\downarrow)$-formula $\psi$ is satisfaction-equivalent to an $L(\downarrow)$-formula $\psi^{*}$ in which independence indications do not cross a $\downarrow$-sign and in which $\sim$ may only occur immediately before $\downarrow$ and in the matrix formula. Replacing in $\psi^{*}$ first all occurrences of $\sim \downarrow$ by $\rightarrow$ and then eliminating in the resulting string all occurrences 
of $\downarrow$, we obtain an $L_{1}$-formula satisfaction-equivalent to $\psi$. Therefore $L(\downarrow) \leq L_{1}$. This allows us to conclude, in view of Theorem 7.2, that actually $L(\downarrow)<\Delta_{2}^{1}$.

The game-based semantics of [5] is interesting as a game-theoretical analysis of Hodges's compositional semantics and more generally in relation to problems due to renaming of variables of IF-logical formulas. The games employed are relatively far removed from Hintikka's GTS: they involve more than two players, and the evaluation procedures carried out by player III are rather complicated. Our metagame analysis has its own complications, but the games remain 2-player games. In the context of $L_{1}$ they provide a game-theoretical analysis of classical negation and offer a way of reformulating Hintikka's subgame notion, which precisely turns on the idea that the strategy functions a player uses in later subgames may take as arguments the adversary's strategy functions in earlier subgames. When moving beyond $L_{1}$, the connective $\rightarrow$ no longer corresponds to classical negation. So, we have found a game-theoretical conceptualization which makes sense in the context of the whole of $L_{2}$ but captures classical negation only in $L_{1}$. Future research will help to assess the general interest of the ideas used to define logic $L_{2}$.

9.4 Negation as a model-theoretic operation Burgess [1] showed (in the equivalent context of Henkin quantifier sentences) that for any mutually incompatible $L_{\mathrm{IF}}$-sentences $\varphi$ and $\psi$ there is a sentence $\theta$ such that $\varphi$ and $\theta$ are truth-equivalent and so are $\psi$ and $\sim \theta$. Kontinen and Väänänen [18] generalized this result to arbitrary formulas of dependence logic. As Burgess noted, it follows that the dual negation fails to correspond to a semantic operation on classes of models: $\sim \theta$ and $\sim \theta^{\prime}$ may even be incompatible while $\theta$ and $\theta^{\prime}$ are truth-equivalent. Because $\sim$ may be eliminated from prefixes of $L_{1}$-formulas, in this logic we need only model-theoretically well-behaved connectives. However, in the context of $L_{2}$ the connective $\rightarrow$ no longer expresses a semantic operation. If, for example, $\varphi:=\exists x \rightarrow(\exists y /\{\exists x\}) R(x, y)$ and $\psi:=\exists x \rightarrow \exists y R(x, y)$, then $\varphi$ and $\psi$ are truth-equivalent (the independence indication only restricts strategies of player $I$ ), though the sentences $\rightarrow \varphi$ and $\rightarrow \psi$ are not.

If we wanted to extend our framework so as to capture stronger fragments of higher-order logic, we might consider introducing a hierarchy of modes in the games and a hierarchy of negations $\rightarrow_{n}$ in the syntax. The negation $>_{n}$ would be interpreted via "metagames of degree $n$," in which depending on the mode one of the players would select a tuple of "local strategy functions of degree $n$," the adversary responding by a tuple of local strategy functions of degree $n-1$. Local strategy functions of degree $n$ would be strategy functions simpliciter in metagames of degree $n-1$. We conjecture that with $n$ negations, we would capture the $\Sigma_{1}^{n+1}$-fragment of $(n+2)$ th-order logic and therefore obtain in particular the full expressivity of $(n+1)$ th-order logic; here only one of the negations (i.e., $\rightarrow n)$ would be modeltheoretically ill-behaved. Developing this generalization is, however, left for another occasion.

\section{Notes}

1. When positions are formulated as above, the component $\epsilon_{i}$ keeps track of the local change, if any, in the overall variable assignment: after the initial position, $\epsilon_{i}$ is always 
empty or a singleton. In this way we avoid encoding in a position more information than needed.

2. Unlike in $L_{0}$ (and even in $L_{\mathrm{IF}}$ ), in $L_{1}$ and $L_{2}$ there being a w.s. for $I$ in $G(\varphi, \mathcal{M}, \gamma,+$ ) is not equivalent to there being a w.s. for $I I$ in $G(\sim \varphi, \mathcal{M}, \gamma,+)$.

3. Actually Hodges formulated the semantics to slash logic (see Hodges [15]), in which quantifiers are of the form $(\mathrm{Q} x / V)$, where $V$ a set of variables (not quantifiers) and variables in $V$ may be "bound" by quantifiers of either force. His semantics can easily be adapted to $L_{\mathrm{IF}}$. It suffices to redefine the equivalence relation among assignments that is used to formulate the semantic clause for slashed existential quantifiers.

\section{References}

[1] Burgess, J. P., "A remark on Henkin sentences and their contraries," Notre Dame Journal of Formal Logic, vol. 44 (2003), pp. 185-88. Zbl 1071.03023. MR 2130790. DOI 10.1305/ndjfl/1091030856. 496

[2] Caicedo, X., F. Dechesne, and T. M. V. Janssen, "Equivalence and quantifier rules for logic with imperfect information," Logic Journal of the IGPL, vol. 17 (2009), pp. 91-129. Zbl 1160.03007. MR 2472294. DOI 10.1093/jigpal/jzn030. 469

[3] Carlson, L. and J. Hintikka, "Conditionals, generic quantifiers, and other applications of subgames" pp. 179-214 in Game-Theoretical Semantics, edited by E. Saarinen, vol. 5 of Synthese Language Library, Reidel, Dordrecht, Netherlands, 1978. 494

[4] Enderton, H. B., "Finite partially-ordered quantifiers," Zeitschrift für mathematische Logik und Grundlagen der Mathematik, vol. 16 (1970), pp. 393-97. Zbl 0193.29405. MR 0284317. 484

[5] Figueira, S., D. Gorín, and R. Grimson, "On the formal semantics of IF-like logics," Journal of Computer and System Sciences, vol. 76 (2010), pp. 333-46. Zbl 1197.03028. MR 2664354. DOI 10.1016/j.jcss.2009.10.006. 495, 496

[6] Figueira, S., D. Gorín, and R. Grimson, "On the expressive power of IF-logic with classical negation," pp. 135-45 in Logic, Language, Information and Computation, edited by L. Beklemishev and R. de Queiroz, Springer, Berlin, 2011. 493, 495

[7] Hintikka, J., "Language-games for quantifiers" pp. 46-72 in Studies in Logical Theory, edited by N. Rescher, Basil Blackwell, Oxford, 1968. 469

[8] Hintikka, J., The Principles of Mathematics Revisited, with an appendix by G. Sandu, Cambridge Univ. Press, Cambridge, 1996. MR 1410063. DOI 10.1017/CBO9780511624919. 470, 473, 481

[9] Hintikka, J., "Negation in logic and in natural language," Linguistics and Philosophy, vol. 25 (2002), pp. 585-600. 470, 493

[10] Hintikka, J., "Truth, negation and other basic notions of logic" pp. 195-219 in The Age of Alternative Logics: Assessing Philosophy of Logic and Mathematics Today, edited by J. van Benthem, et al., Springer, Berlin, 2006. 470, 473, 493, 494

[11] Hintikka, J., and J. Kulas, The Game of Language: Studies in Game-Theoretical Semantics and Its Applications, vol. 22 of Synthese Language Library, D. Reidel, Dordrecht, Netherlands, 1983. MR 0729683. DOI 10.1007/978-94-010-9847-2. 494

[12] Hodges, W., "Compositional semantics for a language of imperfect information," Logic Journal of the IGPL, vol. 5 (1997), pp. 539-63. Zbl 0945.03034. MR 1465612. DOI 10.1093/jigpal/5.4.539. 494, 495

[13] Hodges, W., "Some strange quantifiers," pp. 51-65 in Structures in Logic and Computer Science, edited by J. Mycielski, G. Rozenberg, and A. Salomaa, vol. 1261 
of Lecture Notes in Computer Science, Springer, Berlin, 1997. MR 1638352. DOI 10.1007/3-540-63246-8 4. 494

[14] Hodges, W., "Elementary predicate logic" pp. 1-129 in Handbook of Philosophical Logic, Vol. 1, edited by D. M. Gabbay and F. Guenthner, Kluwer, Dordrecht, Netherlands, 2001. MR 1884623. 470

[15] Hodges, W., "Logics of imperfect information: Why sets of assignments?" pp. 117-33 in Interactive Logic, edited by J. van Benthem, B. Löwe, and D. Gabbay, vol. 1 of Texts in Logic and Games, Amsterdam Univ. Press, Amsterdam, 2007. MR 2508176. 497

[16] Janssen, T. M. V., "Independent choices and the interpretation of IF logic: Logic and games," Journal of Logic, Language and Information, vol. 11 (2002), pp. 367-87. Zbl 1003.03025. MR 1909620. DOI 10.1023/A:1015542413718. 495

[17] Kontinen, J., and V. Nurmi, "Team logic and second-order logic," Fundamenta Informaticae, vol. 106 (2011), pp. 259-72. Zbl 1250.03048. MR 2810064. 494

[18] Kontinen, J., and J. Väänänen, "A remark on negation in dependence logic," Notre Dame Journal of Formal Logic, vol. 52 (2011), pp. 55-65. Zbl 1216.03048. MR 2747162. DOI 10.1215/00294527-2010-036. 496

[19] Krynicki, M., "Henkin quantifiers" pp. 193-262 in Quantifiers: Logics, Models and Computation, Vol. 1, edited by M. Krynicki, M. Mostowski, and L. Szczerba, Kluwer, Dordrecht, Netherlands, 1995. 484

[20] Mostowski, M., "Arithmetic with the Henkin Quantifier and its Generalizations," pp. 1-25 in Séminaire du Laboratoire Logique, Algorithmique et Informatique Clermontois, Vol. 2, edited by F. Gaillard and D. Richard, Institut universitaire de technologie de Clermont-Ferrand, Aubière, 1991. 484

[21] Väänänen, J., Dependence Logic: A New Approach to Independence Friendly Logic, vol. 70 of London Mathematical Society Student Texts, Cambridge Univ. Press, Cambridge, 2007. MR 2351449. DOI 10.1017/CBO9780511611193. 494

[22] Walkoe, W., "Finite partially-ordered quantification," Journal of Symbolic Logic, vol. 35 (1970), pp. 535-55. Zbl 0219.02008. MR 0278920. 484

\section{Acknowledgment}

I wish to express my gratitude to the anonymous referees whose comments and criticism helped to improve the paper.

CNRS research unit "Savoirs, Textes, Langage" and

Department of Philosophy, University of Lille 3

Domaine Universitaire du "Pont de Bois"

B.P. 149, 59653 Villeneuve d'Ascq

France

tero.tulenheimo@univ-lille3.fr

http://www.tulenheimo.webs.com 